Preprint typeset in JHEP style - HYPER VERSION

\title{
Warped Radion Inflation
}

\author{
Joel Trudeau, James M. Cline \\ Department of Physics, McGill University, Montréal, QC, H3A 2T8, Canada \\ trudeau@physics.mcgill.ca, jcline@physics.mcgill.ca
}

\begin{abstract}
We show that the radion in a warped geometry bounded by two branes can have a potential suitable for inflation. Our construction is based upon a solution known in string theory as the linear dilaton, in which the back-reaction from a bulk scalar $\Phi$ is exactly accounted for. The radion, stabilized by $\Phi$, is much heavier than the TeV scale and its couplings to the standard model are much more suppressed than in the usual RandallSundrum solution. We present a new formalism for obtaining approximate time-dependent solutions, based on perturbing the exact solution to the coupled Einstein and scalar field equations in the bulk. It allows the radion potential to be computed directly in terms of the brane potentials for $\Phi$. We show that simple exponential potentials on the branes can lead to a $4 \mathrm{D}$ radion potential with a flattened hilltop form, yielding inflation with a spectral index of typically $n_{s}=0.96$ and no higher than 0.99 . With more complicated brane potentials, the descent from the hilltop can be a linear potential, giving a tensor-to-scalar ratio as large as $r=0.07$ with $n_{s}=0.974$. The couplings of the radion to the standard model particles are dictated by general covariance, so the details of reheating are explicitly calculable, leading to a reheat temperature of at least $10^{7} \mathrm{GeV}$. The quantum corrections to the inflaton potential from its couplings to matter are also calculable and are shown to be small, so that the prediction for the shape of the potential is under theoretical control, even with superPlanckian field excursions.
\end{abstract}




\section{Contents}

1. Introduction 1

2. Unperturbed static solution 3

2.1 Static solutions 3

2.2 Junction conditions 5

2.3 Exponential brane potentials 6

3. Time-dependent solutions 6

3.1 Small perturbations with large radion fluctuations

3.2 The $G_{05}$ equation and junction conditions 8

3.3 The remaining Einstein equations 11

4. Inflation in the effective 4D theory 13

4.1 Radion stability 14

4.2 Kaluza-Klein excitations

4.3 Radion inflation: an explicit model

4.4 Model with larger tensors

4.4.1 Analytic treatment 22

4.4 .2 Numerical analysis 24

5. Coupling of radion to standard model 25

5.1 Reheating [25]

5.2 Radiative corrections to inflaton potential 26

6. Discussion 27

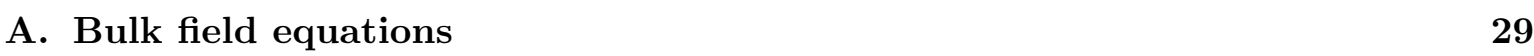

B. Kaluza-Klein excitations $\quad 29$

\section{Introduction}

The possibility that we live on a brane embedded in a higher-dimensional space has been widely explored since one of its earliest string theory manifestations, the Horava-Witten model [1]. Brane-worlds afford intriguing opportunities to go beyond the standard model of particle physics and cosmology, but they also present challenges for reproducing known physics. The 
existence of moduli in these models is a generic feature with potentially important cosmological consequences. If unstabilized, they can conflict with big bang nucleosynthesis or fifth force constraints. This was the case in the original proposal of Randall and Sundrum (RS1) [2], which elegantly addressed the weak-scale hierarchy problem. These issues were resolved by introducing a bulk scalar field to stabilize the radion, the modulus determining the size of the extra dimension $[3]$.

Moduli are natural candidates to serve as the inflaton, and the radion is foremost among them. However it has been difficult to make radion inflation work in practice. Ref. 沺 proposed the modulus of large extra dimensions [5] as the inflaton, but this required an inflaton potential with a rather peculiar shape to have inflation at the TeV scale [6]. In the framework of warped extra dimensions, most of the effort has been either on inflation driven by motion of branes within the bulk, or else conventional inflation on one of the branes modified by an unconventional Friedmann equation due to the extra dimensions. We are not aware of previous proposals for getting inflation from the radion in the RS-I (orbifold bounded by two branes) scenario. ${ }^{1}$ It would be difficult to make this work, first because the Goldberger-Wise mechanism for stabilizing the radion does not give it a flat potential, and secondly because at Planck-scale values of the canonically normalized radion field, which could in principle have given chaotic inflation, the radion has already passed the barrier in the potential separating the compactified and decompactified regimes of the theory. In fact the light KK modes of the extra dimension become excited, invalidating the $4 \mathrm{D}$ effective description, long before the radion can reach the Planck scale.

In this work we present a model of a warped $5 \mathrm{D}$ radion as inflaton that overcomes these difficulties. We incorporate a warped background solution that has negative curvature but is not AdS; instead it is conformally flat, and relies on a linearly varying bulk scalar field for the source of its negative bulk energy density. The weak scale hierarchy problem is naturally solved, and inflation occurs at a high scale, with weak couplings of the inflaton to matter on the standard model brane. We are able to relate the potential of the radion to the bulk scalar field potentials on the branes in a rather simple way, and to find radion potentials (starting from brane potentials of exponential form) that are flat enough for inflation with a certain amount of tuning of parameters of the brane potentials.

Generically, one always expects decompactified flat space to be a solution to the Einstein equations, and so the radion potential naturally has degenerate minima representing compact and noncompact fifth dimension, separated by a barrier. In our model the barrier is at Planckian field values, and we can find examples where superPlanckian excursions during inflation lead to an observable tensor component in the perturbations. Other examples have a small tensor component with inflation ending close to the hilltop. Because the minima are degenerate, we have the situation of topological inflation [8, 9]: the universe divides into 4D and 5D domains separated by eternally inflating domain walls where the radion is near the top of the potential. This guarantees the existence of regions where the inflaton is arbitrarily

\footnotetext{
${ }^{1}$ a recent reference using different moduli fields is $[$ ].
} 
close to the potential maximum (up to the limitation imposed by quantum fluctuations of the field) so that one does not have to rely upon fine-tuning of the initial position of the inflaton to get enough inflation.

In section 2 we introduce the static solution with stabilized radion and contrast it to the RS solution, especially with regard to the hierarchy problem. In section 3 we perturb away from the static solution to find time-dependent ones, in the approximation that the back-reaction is small. We derive the effective $4 \mathrm{D}$ theory in section 1 , computing the mass of the radion and the spectrum of Kaluza-Klein excitations for the radion and bulk scalar. We then construct some inflationary models using exponential brane potentials and work out their predictions for the CMB power spectrum, using both analytic and numerical techniques. Section 5 derives the couplings of the radion to the standard model, enabling us to compute the reheat temperature at the end of inflation and to estimate the radiative corrections to the inflaton potential. We give conclusions in section 6 and the bulk Einstein and scalar field equations in the appendix.

\section{Unperturbed static solution}

We begin by introducing the framework for our 5D brane-world model. The action for 5D gravity coupled to the stabilizing scalar field, $\Phi=\Phi(y, t)$ with a bulk potential $V(\Phi)$ and potentials on the branes $V_{0,1}(\Phi)$ is

$$
\begin{gathered}
S=2 \int_{0}^{1} d^{5} x \sqrt{g}\left(-\frac{1}{2 \kappa_{5}^{2}} R+\frac{1}{2} \partial^{\mu} \Phi \partial_{\mu} \Phi-V(\Phi)\right)-\left.\int d^{4} x \sqrt{g_{4}} V_{0}\right|_{y=0}-\left.\int d^{4} x \sqrt{g_{4}} V_{1}\right|_{y=1} \\
-\left.\kappa_{5}^{2} \int d^{4} x \sqrt{g_{4}}[K]\right|_{y=0}-\left.\kappa_{5}^{2} \int d^{4} x \sqrt{g_{4}}[K]\right|_{y=1}
\end{gathered}
$$

The extra dimension is an $S^{1} / Z_{2}$ orbifold (hence the factor of 2 ) and all functions are symmetric under $y \rightarrow-y$. Thus we consider the interval $y \in[0,1]$ for the compact extra dimension with the Planck $\left(y_{0}=0\right)$ and $\mathrm{TeV}\left(y_{1}=1\right)$ branes located at the orbifold fixed points. We also include extrinsic curvature terms $[K]$ that are needed for properly defining the variations of the action.

The most general metric ansatz that respects $3 \mathrm{D}$ homogeneity and isotropy can be written as

$$
d s^{2}=e^{2 N(t, y)} d t^{2}-e^{2 A(t, y)} d \mathbf{x}^{2}-e^{2 B(t, y)} d y^{2}
$$

The field equations for this ansatz are well known and we reproduce them in appendix $\mathrm{A}$.

\subsection{Static solutions}

It will be useful for what follows to construct an exact solution to the coupled bulk scalar field and Einstein equations, which is conformally flat:

$$
d s^{2}=e^{2 n(y)}\left(d t^{2}-d \mathbf{x}^{2}-b^{2} d y^{2}\right)
$$

Notice that this differs from $\mathrm{AdS}_{5}$ in Randall-Sundrum coordinates where the $e^{2 n(y)}$ factor does not multiply $d y^{2}$. The negative vacuum energy density provided by the bulk scalar 
potential is not constant, so the geometry is not AdS. This solution is well-known (see e.g., [10]- [12]) in the context of strings propagating in a spacetime with subcritical dimensionality, compensated by a spatially linearly varying dilaton similar to our bulk scalar. With this ansatz the Einstein equations in the bulk reduce to

$$
\begin{aligned}
-3\left(n^{\prime \prime}+n^{\prime 2}\right) & =\kappa_{5}^{2}\left(\frac{1}{2} \Phi^{\prime 2}+b^{2} e^{2 n} V(\Phi)\right) \\
-6\left(n^{\prime}\right)^{2} & =\kappa_{5}^{2}\left(-\frac{1}{2} \Phi^{\prime 2}+b^{2} e^{2 n} V(\Phi)\right)
\end{aligned}
$$

As usual, the scalar field equation of motion

$$
\Phi^{\prime \prime}+3 n^{\prime} \Phi^{\prime}-b^{2} e^{2 n} \frac{d V}{d \Phi}=0
$$

is not independent of the Einstein equations, but can be obtained by taking linear combinations of (2.4, 2.5) and derivatives thereof. By choosing the bulk potential to take a special form, $V=-\bar{V} \exp \left(c_{V} \Phi\right)$, one finds that the solutions for $n$ and $\Phi$ are simple linear functions of $y: n(y)=n_{0}-k b y, \Phi(y)=\Phi_{0}+c_{\Phi} y$. Here $k$ plays the role of the AdS curvature scale, as in the usual RS solution, and by analogy we will so refer to it, even though the solution is not AdS. The integration constants $n_{0}$ and $\Phi_{0}$ can be absorbed into the normalizations of $b$ and $\bar{V}$ respectively without loss of generality, so we set them to zero. The equations of motion then imply that $\bar{V}=-\frac{9 k^{2}}{2 \kappa_{5}^{2}}, c_{V}=2 \frac{\kappa_{5}}{\sqrt{3}}, c_{\Phi}=\frac{\sqrt{3} k b}{\kappa_{5}}$. With these choices, the bulk scalar potential is given by

$$
V(\Phi)=-\frac{3}{2} \mu_{5}^{2} k^{2} \exp \left(2 \mu_{5}^{-1} \Phi\right)
$$

where we define

$$
\mu_{5}=\frac{\sqrt{3}}{\kappa_{5}}
$$

and the solution is

$$
n(y)=-k b y ; \quad \Phi=\mu_{5} k b y
$$

In contrast to the original RS construction, we do not include a bulk cosmological constant; the negative bulk potential $V(\Phi)$ is responsible for the curvature of the $5 \mathrm{D}$ geometry. Similar negative potentials have been studied in the context of Golberger-Wise stabilization of bulk fields in $\mathrm{AdS}_{5}$ [13], where it was observed that the unboundedness of the potential does not lead to instabilities in the AdS background [14]. The present case is similar; in the analogous subcritical string theory situation where the bulk scalar is the dilaton, in the string frame the negative coefficient of the potential is interpreted as a negative bulk cosmological constant, which would give rise to an AdS background if the dilaton were held fixed. In the present situation we have in addition the couplings of the bulk field to the branes, which prevent the field from running away. 


\subsection{Junction conditions}

The Israel junction conditions (see e.g., ref. [15]) and the boundary conditions for the scalar field are given by

$$
\left.b^{-1} e^{-n} n^{\prime}\right|_{y_{i}-\epsilon} ^{y_{i}+\epsilon}= \pm\left.\frac{\kappa_{5}^{2} V_{i}}{3}\right|_{y_{i}},\left.\quad b^{-1} e^{-n} \Phi^{\prime}\right|_{y_{i}-\epsilon} ^{y_{i}+\epsilon}= \pm\left.\frac{\partial V_{i}}{\partial \Phi}\right|_{y_{i}}
$$

where the \pm apply respectively at $y_{0}=0$ and $y_{1}=1$. Then explicitly,

$$
\begin{aligned}
2 \mu_{5}^{2} k & =V_{0}(0), & 2 \mu_{5}^{2} k & =-e^{n_{1}} V_{1}\left(\Phi_{1}\right) \\
2 \mu_{5} k & =\frac{\partial V_{0}}{\partial \Phi}(0), & 2 \mu_{5} k & =-e^{n_{1}} \frac{\partial V_{1}}{\partial \Phi}\left(\Phi_{1}\right)
\end{aligned}
$$

where $e^{n_{1}}=e^{-k b}$ and $\Phi_{1}=\mu_{5} k b$ are respectively the warp factor and the scalar field value on the $\mathrm{TeV}$ brane.

Let us recall the physical significance of the junction conditions. In the pure RS model with no scalar field, the two junction conditions require fine-tuning of the brane tensions (here represented by the values of the potentials $V_{0}$ and $V_{1}$ on the branes). For generic values of the tensions, one would obtain a bulk solution that is not static, and which contains a black hole in the extra dimension [16]. Thus the two tunings can be interpreted as (1) the usual setting of the 4D cosmological constant to zero, to obtain a static solution, and (2) the tuning of the bulk black hole mass to zero. The radion $b$ is exactly massless in this solution, and can thus take any value.

Next consider the addition of the bulk scalar field. In the usual implementations of the Goldberger-Wise mechanism, the back-reaction of the scalar field on the metric is taken to be small, and one simply solves the bulk scalar equation in the $\mathrm{AdS}_{5}$ background. This is a second order equation, and the two constants of integration in the solution are determined by the two additional boundary conditions involving $d V_{i} / d \Phi$. This generically induces a potential for the radion that is minimized at some value $b_{0}$.

In our solution, the situation is somewhat different; we have singled out a particularly simple form of the scalar field solution, which is only compatible with certain choices of $d V_{i} / d \Phi$ at the boundaries. It is still true that the radion is stabilized as in the generic $\mathrm{GW}$ mechanism, but there is an additional tuning of potential parameters needed to maintain the linear solution for $\Phi$. This extra tuning is on the same footing as that of the bulk black hole mass in the pure RS solution. We suspect that our results can be generalized to more complicated solutions in which this tuning is relaxed, but for ease of computation, we will adhere to this special situation.

The upshot is that the junction conditions amount to three tunings of brane potential parameters, plus one condition that fixes the value of the radion $b$, and hence the warp factor on the TeV brane. Let us illustrate with a simple example of brane potentials which is similar to that made by GW:

$$
V_{0}=m_{0}^{2}\left(\Phi+u_{0}\right)^{2}, \quad V_{1}=-m_{1}^{2}\left(\Phi+u_{1}\right)^{2}
$$


Solving the junction conditions, we obtain three constraints that can be regarded as finetunings on the parameters $m_{0}, u_{0}$ and $u_{1}$,

$$
m_{0}^{2}=\frac{k}{2}, \quad u_{0}=2 \mu_{5}, \quad u_{1}=\mu_{5}(2-k b)
$$

while $m_{1}$ can be regarded as adjustable, and its value determines the warp factor through

$$
e^{-k b_{0}}=\frac{m_{0}^{2}}{m_{1}^{2}}
$$

One notices a disadvantage of our approach relative to the usual one; we have had to build the hierarchy of scales into our original choice of brane couplings to get an exponentially small warp factor, as opposed to having generic Planck-scale values for the brane potential parameters. The next example shows that this problem can be ameliorated if the potentials take an exponential form.

\subsection{Exponential brane potentials}

In our subsequent construction of an inflationary solution, we will make use of a different choice of brane potentials, which are exponentials in $\Phi$ :

$$
\begin{aligned}
& V_{0}=\Lambda_{0} e^{\alpha_{0} \Phi / \mu_{5}}-\Delta_{0} e^{\beta_{0} \Phi / \mu_{5}} \\
& V_{1}=-\Lambda_{1} e^{\alpha_{1} \Phi / \mu_{5}}+\Delta_{1} e^{\beta_{1} \Phi / \mu_{5}}
\end{aligned}
$$

The junction conditions lead to

$$
\begin{array}{ll}
\Lambda_{0}=2 \mu_{5}^{2} k\left(\frac{1-\beta_{0}}{\alpha_{0}-\beta_{0}}\right), & \Delta_{0}=2 \mu_{5}^{2} k\left(\frac{1-\alpha_{0}}{\alpha_{0}-\beta_{0}}\right) \\
\Lambda_{1}=2 \mu_{5}^{2} k\left(\frac{1-\beta_{1}}{\alpha_{1}-\beta_{1}}\right) e^{\left(1-\alpha_{1}\right) k b_{0}}, & \Delta_{1}=2 \mu_{5}^{2} k\left(\frac{1-\alpha_{1}}{\alpha_{1}-\beta_{1}}\right) e^{\left(1-\beta_{1}\right) k b_{0}}
\end{array}
$$

For a given desired value of $b_{0}$ determining the hierarchy of scales between the two branes, there are thus four free parameters $\alpha_{i}, \beta_{i}$ that can eventually be used to tune the potential of the radion to a form suitable for inflation, while the dimensionful couplings $\Delta_{i}$ and $\Lambda_{i}$ are fixed in terms of these. Notice that if $\alpha_{1}$ and $\beta_{1}$ happen to be moderately close to 1 (e.g., $\sim 1.1$ ), the explicit hierarchy can be ameliorated between the dimensionful parameters on the Planck brane versus those on the TeV, while yielding a sufficiently large value of $k b_{0} \sim 37$ to explain the TeV scale, since then $\Lambda_{1} / \Lambda_{0} \sim e^{\left(1-\alpha_{1}\right) k b_{0}}$ and $\Delta_{1} / \Delta_{0} \sim e^{\left(1-\beta_{1}\right) k b_{0}}$.

\section{Time-dependent solutions}

Our goal now is to extend the static solution of the previous section to dynamical ones in which the radion is displaced from its stable equilibrium value $b_{0}$. In refs. [15], [17]- [20], 5D solutions that also include the dynamics of a bulk scalar field were found for the case of a single brane. In these papers the search for solutions was simplified by assuming that the 
metric functions and the bulk scalar were separable functions of $t$ and $y$, which we will justify. The key to our approach will be to allow for a large excursion of the radion field (which plays the role of the inflaton) in response to linear perturbations of the metric and bulk scalar. Although such an ansatz is not guaranteed to be consistent, we will show that for suitable choices of the parameters for the exponential brane potentials (2.16,2.17) it is justified, and will allow us to find physically interesting inflationary solutions.

\subsection{Small perturbations with large radion fluctuations}

We make an ansatz for general scalar perturbations similar to that in ref. [21,

$$
\begin{aligned}
d s^{2} & =e^{2 n(y)}\left[e^{2 F(x, y)} \eta_{\mu \nu} d x^{\mu} d x^{\nu}-b_{0}^{2} e^{2 \varphi(t)+2 G(x, y)} d y^{2}\right] \\
\Phi(x, y) & =-\mu_{5}(n(y)+\varphi(t))+\delta \Phi(x, y)
\end{aligned}
$$

It reduces to the static solution of the previous section when $F=G=\delta \Phi=\varphi(t)=0$. Normally then, one would linearize in all of these quantities. However we will find a more general solution for the perturbations by treating only $F, G, \delta \Phi$ as being small, while working to all orders in $\varphi(t)$ (and $\dot{\varphi}$ ). This corresponds to allowing for large excursions of the radion during inflation. We will justify it a postieriori by choosing special values of the brane potentials that lead to a sufficiently flat inflaton potential.

The perturbations $F, G, \delta \Phi$ are constrained by the nondynamical Einstein equations, namely those containing only first derivatives. The $G_{05}$ equation gives

$$
\left(\dot{G}+\mu_{5}^{-1} \dot{\delta \Phi}\right) n^{\prime}+\left(F^{\prime}+\mu_{5}^{-1} \delta \Phi^{\prime}\right) \dot{\varphi}-\dot{F}^{\prime}=0
$$

Similarly the off-diagonal $G_{i j}$ equation gives

$$
2 \partial_{i} \partial_{j} F+\partial_{i} \partial_{j} G=0
$$

It can be integrated to find

$$
G(x, y)=-2 F(x, y)+G_{0}(t, y)
$$

where an untenable term linear in $\mathbf{x}$ has been set to zero. The $G_{0 j}$ equation

$$
\left(\partial_{j} F+\mu_{5}^{-1} \partial_{j} \delta \Phi\right) \dot{\varphi}=0
$$

can be solved for the bulk scalar perturbations, using eq. (3.5) :

$$
\delta \Phi(x, y)=-\mu_{5} F(x, y)+\delta \Phi_{0}(t, y)
$$

Lastly the $G_{i 5}$ equation, after substituting eqs. (3.5) and (3.7), reduces to

$$
\partial_{j}\left(F^{\prime}+3 n^{\prime} F\right)=0
$$


The general solution to $(3.8)$ is

$$
F(x, y)=\hat{F}(x) e^{-3 n(y)}+F_{0}(t, y)
$$

We find that the $\hat{F}(x)$ terms in the solutions to the metric and scalar field perturbations, eqs. (3.5, 3.7, 3.9), vanish at linear order from the off-diagonal constraint equations and so $\hat{F}(x)$ is undetermined. Therefore it can be consistently set to zero.

Now let us specialize the general ansatz to a form that is adapted for the cosmological solutions we seek:

$$
\begin{aligned}
d s^{2} & =e^{2 n(y)}\left[e^{2 F_{1}(\varphi(t), y)+2 F_{2}(\varphi(t), y)}\left(d t^{2}-a^{2}(t) d \mathbf{x}^{2}\right)-b_{0}^{2} e^{2 \varphi(t)-4 F_{2}(\varphi(t), y)} d y^{2}\right] \\
F_{1}(\varphi, y) & =n(y) f(\varphi(t)), \quad F_{2}(\varphi, y)=f_{2}(\varphi(t)) e^{(3+\varepsilon)\left(n_{1}-n(y)\right)} \\
\Phi(t, y) & =\mu_{5}\left(k b_{0} y-\varphi(t)\right)+\delta \Phi(\varphi(t), y)
\end{aligned}
$$

where $n_{1}=n(1)=-k b_{0}$ and $\varepsilon$ is an adjustable parameter. ${ }^{2}$ It reduces to the previous static solution when the radion fluctuation $\varphi(t)$ vanishes, if $f, f_{2}$ and $\delta \Phi$ also vanish. One can interpret $f, f_{2}$ and $\delta \Phi$ as small back-reactions induced on the metric and scalar field, respectively, by the (possibly large) radion fluctuation $\varphi$. This ansatz does not provide an exact solution to the equations of motion, but it is a good approximation at leading order in $f, f_{2}$ and $\delta \Phi$ as long as these quantities are small, in a sense to be specified. We will find situations where it is possible to reliably consider large excursions of the radion during inflation, even superPlanckian ones. It will be shown that the functions $f, f_{2}$ and $\delta \Phi$ can be algebraically derived from the brane potentials $V_{0}$ and $V_{1}$. In turn they determine the potential for the radion in the $4 \mathrm{D}$ effective theory. We will find that the radion potential derived from this ansatz is dominated by contributions from $f$ and its bulk scalar counterpart (the part that is flat in the extra dimension). The other parts of the perturbations, those with exponential $y$-dependence, turn out to be relevant solely for the stabilization of the static solution.

\subsection{The $G_{05}$ equation and junction conditions}

To arrive at the functional form of the scalar perturbations we examine the off-diagonal Einstein equation. Linearizing the left hand side we find

$$
-\dot{\varphi}\left(1+f-\frac{d f}{d \varphi}-3 F_{2}+\frac{d F_{2}}{d \varphi}\right)=\mu_{5}^{-2} \dot{\Phi} \Phi^{\prime}
$$

This motivates us to parametrize $\delta \Phi(\varphi, y)=\delta \Phi_{1}+\delta \Phi_{2}$ where

$$
\mu_{5}^{-1} \delta \Phi_{1}(\varphi, y)=g(\varphi), \quad \quad \mu_{5}^{-1} \delta \Phi_{2}(\varphi, y)=g_{2}(\varphi) e^{(3+\varepsilon)\left(n_{1}-n(y)\right)}
$$

This separation is useful, because it will be necessary to require that $f, f_{2}, g, g_{2} \ll 1$ but not necessarily that $\varphi \ll 1$. With this parametrization, the dominant " 1 " terms which are zeroth

\footnotetext{
${ }^{2}$ We will see that the choice $\epsilon=-1$ leads to small back-reaction of $f, f_{2}$ and $\delta \Phi$ while allowing for large $\varphi$
} 
order in the perturbations cancel as in our previous analysis and the linearized $G_{05}$ equation becomes

$$
-f+\frac{d f}{d \varphi}-\frac{d g}{d \varphi}=e^{(3+\varepsilon) k\left(y-b_{0}\right)}\left((1+\varepsilon) \frac{d f_{2}}{d \varphi}+\frac{d g_{2}}{d \varphi}-(3+\varepsilon)\left(f_{2}+g_{2}\right)\right)
$$

It is satisfied everywhere in the bulk if

$$
\begin{gathered}
\frac{d f}{d \varphi}-\frac{d g}{d \varphi}=f \\
(1+\varepsilon) \frac{d f_{2}}{d \varphi}+\frac{d g_{2}}{d \varphi}=(3+\varepsilon)\left(f_{2}+g_{2}\right)
\end{gathered}
$$

and has negligible $y$-dependence when

$$
f_{2}, g_{2} \ll \varphi \quad \text { and } \quad f_{2}, g_{2} \ll 1
$$

In our numerical analysis we find eqs. (3.16, 3.17) hold to a high degree of accuracy when the back-reaction is small. Moreover, for the cases we study, eq. (3.17) will reduce to eq. (3.16).

Now let us reconsider the junction conditions in the presence of the perturbations:

$$
\begin{aligned}
\left.2 \mu_{5}^{2} k\left(1+f-(1+\varepsilon) f_{2} e^{-(3+\varepsilon)\left(n_{i}-n_{1}\right)}\right) e^{-n_{i}-\varphi}\right|_{y=y_{i}} & = \pm\left. V_{i}\left(\Phi_{i}+\delta \Phi\right)\right|_{y=y_{i}} \\
\left.2 \mu_{5} k\left(1+\left((3+\varepsilon) g_{2}+2 f_{2}\right) e^{-(3+\varepsilon)\left(n_{i}-n_{1}\right)}\right) e^{-n_{i}-\varphi}\right|_{y=y_{i}} & = \pm\left.\frac{\partial V_{i}}{\partial \Phi}\left(\Phi_{i}+\delta \Phi\right)\right|_{y=y_{i}}
\end{aligned}
$$

where now $\Phi_{0}=-\mu_{5} \varphi$ and $\Phi_{1}=\mu_{5}\left(k b_{0}-\varphi\right)$ take the place of the unperturbed scalar field at $y=0$ and $y=1$. These are algebraic equations that determine the back-reaction functions $f, g$ and $f_{2}, g_{2}$. At low energies the functions all have a linear dependence on $\varphi$ and are related as $g=f$ and $g_{2}=(1+\varepsilon) f_{2}$. However, when $\varepsilon=-1, g_{2}$ vanishes at linear order in $\varphi$ so that the bulk scalar perturbations are effectively independent of $y$. Even when $\varphi$ is large, this choice simplifies the subsequent analysis without affecting the radion dynamics, so we will adopt $\varepsilon=-1$ from now on.

The linearized jump conditions can be solved to first order in $g$ by Taylor-expanding $V_{0}^{\prime}$ about $\Phi=-\mu_{5} \varphi$ at $y=0$ :

$$
g \cong-g_{2} e^{2 n_{1}}+\frac{2 k e^{-\varphi}\left(1+\left(2 f_{2}+2 g_{2}\right) e^{2 n_{1}}\right)-\mu_{5}^{-1} V_{0}^{\prime}}{V_{0}^{\prime \prime}}
$$

where the prime on potentials denotes $\frac{d}{d \Phi}$. Expanding next for $V_{0}$ in eq. (3.19) we find

$$
f=-1+\frac{e^{\varphi}}{2 \mu_{5}^{2} k}\left(V_{0}+\mu_{5}\left(g+g_{2} e^{2 n_{1}}\right) V_{0}^{\prime}\right)
$$

Similarly, at $y=1$, we expand $V_{1}^{\prime}$ about $\Phi=\Phi_{1}-\mu_{5} \varphi$ and find for arbitrary stabilizing potentials using (3.21) and (3.22)

$$
f_{2}=-g_{2}\left[\frac{1}{2}+\frac{V_{0}^{\prime \prime} V_{1}^{\prime \prime} e^{n_{1}} e^{\varphi}\left(1-e^{2 n_{1}}\right)}{4 k\left(V_{0}^{\prime \prime}+V_{1}^{\prime \prime} e^{3 n_{1}}\right)}\right]-\left[\frac{\frac{1}{2}\left(V_{0}^{\prime \prime}+V_{1}^{\prime \prime} e^{n_{1}}\right)+\frac{e^{\varphi}}{4 \mu_{5} k}\left(V_{0}^{\prime \prime} V_{1}^{\prime} e^{n_{1}}-V_{0}^{\prime} V_{1}^{\prime \prime} e^{n_{1}}\right)}{V_{0}^{\prime \prime}+V_{1}^{\prime \prime} e^{3 n_{1}}}\right]
$$


Then expanding for $V_{1}$ in eq. (3.19)

$$
\begin{gathered}
g_{2}=G_{2}^{-1}\left[2 k e^{-\varphi} \mu_{5}\left(V_{0}^{\prime}+V_{1}^{\prime} e^{n_{1}}\right)+\left[e^{2 n_{1}}\left(\left(V_{0}+V_{1} e^{n_{1}}\right) V_{1}^{\prime \prime} e^{n_{1}}-\left(V_{0}^{\prime}+V_{1}^{\prime} e^{n_{1}}\right) V_{1}^{\prime} e^{n_{1}}\right)\right.\right. \\
\left.\left.+\left(V_{0}+V_{1} e^{n_{1}}\right) V_{0}^{\prime \prime}-\left(V_{0}^{\prime}+V_{1}^{\prime} e^{n_{1}}\right) V_{0}^{\prime}\right]\left(1-e^{2 n_{1}}\right)^{-1}\right]
\end{gathered}
$$

where

$$
G_{2}=V_{0}^{\prime} V_{1}^{\prime \prime} e^{3 n_{1}}-V_{1}^{\prime} e^{n_{1}} V_{0}^{\prime \prime}
$$

A fine-tuned choice of brane potentials such that $V_{0}=-V_{1} e^{n 1}$ is tempting for simplifying the solutions since then $g_{2}=f_{2}=0$. However, our study of the KK excitations (section 4.2) shows that this choice is precluded by the presence of a tachyon instability. The expressions for the back-reaction functions may nevertheless be simplified by neglecting the subdominant contributions involving extra powers of the warp factor:

$$
\begin{aligned}
g & \cong \frac{2 k e^{-\varphi}-\mu_{5}^{-1} V_{0}^{\prime}}{V_{0}^{\prime \prime}} \\
f & \cong-1+\frac{e^{\varphi}}{2 \mu_{5}^{2} k}\left(V_{0}+\mu_{5} g V_{0}^{\prime}\right) \\
g_{2} & \cong-g-\frac{2 k e^{-\varphi} \mu_{5}(1+f)+\mu_{5}^{-1} V_{1} e^{n_{1}}}{V_{1}^{\prime} e^{n_{1}}} \\
f_{2} & \cong-g_{2}\left(1+\frac{e^{\varphi}}{4 k} V_{1}^{\prime \prime} e^{n_{1}}\right)-\frac{1}{2}-\frac{e^{\varphi}}{4 k}\left(\mu_{5}^{-1} V_{1}^{\prime} e^{n_{1}}+V_{1}^{\prime \prime} e^{n_{1}} g\right)
\end{aligned}
$$

Our results for $f, g$ and $f_{2}, g_{2}$ are derived from the jump conditions and not from the Einstein equations, so it is not obvious that they satisfy the relation (3.15) from the $G_{05}$ equation. However, it is simple to show that this indeed is the case, at linear order. Differentiating the first jump condition (at $y=0$ or $y=1$ ) in (3.19) with respect to $\varphi$ using (3.14) gives

$$
\left.\frac{d V_{i}}{d \Phi}\right|_{y=y_{i}}=\left.\frac{d \varphi}{d \Phi} \frac{d V_{i}}{d \varphi}\right|_{y=y_{i}}=\left.\mu_{5}\left(-1+\frac{d g}{d \varphi}+\frac{d g_{2}}{d \varphi} e^{-2\left(n-n_{1}\right)}\right)^{-1} \frac{d V_{i}}{d \varphi}\right|_{y=y_{i}}
$$

Eliminating $\frac{d V_{i}}{d \Phi}$ using the second jump condition (at $y=0$ or $y=1$ ) in (3.20) and linearizing, the dominant "1" terms again balance and we arrive directly at (3.15) evaluated on the branes (which also guarantees that (3.15) is satisfied in the bulk since both sides of the equation vanish). This is similar to what was found in ref. 21] where the linearized offdiagonal Einstein equation, $G_{\mu 5}$ evaluated on the branes was shown to be equivalent to one of the junction conditions of the metric so that no new constraints arise.

For small $\varphi \ll 1$ we can Taylor-expand about the background and arrive at simple expressions for the back-reaction functions

$$
\begin{aligned}
g & =f, \quad g=\frac{-\sigma_{0}\left(\varphi-g_{2} e^{-2 k b_{0}}\right)}{1-\sigma_{0}} \simeq-\frac{\sigma_{0} \varphi}{1-\sigma_{0}} \\
g_{2} & =0, \quad f_{2}=\frac{1}{2} \frac{\varphi\left(\sigma_{1}-\sigma_{0}\right)}{\left(1-\sigma_{0}-e^{-2 k b_{0}}\left(1-\sigma_{1}\right)\right)} \simeq \frac{1}{2} \frac{\varphi\left(\sigma_{1}-\sigma_{0}\right)}{\left(1-\sigma_{0}\right)}
\end{aligned}
$$


where the jump conditions

$$
\pm\left.\mu_{5}^{-1} e^{n_{i}} V_{i}^{\prime}(\Phi)\right|_{\Phi_{i}}= \pm\left. e^{n_{i}} \mu_{5}^{-2} V_{i}(\Phi)\right|_{\Phi_{i}}=2 k
$$

have motivated us to parametrize the brane potentials such that

$$
\pm\left. e^{n_{i}} V_{i}^{\prime \prime}(\Phi)\right|_{\Phi_{i}}=2 k\left(1-\sigma_{i}\right)
$$

This has a convenient physical interpretation. When $\sigma_{i}=0$ the radion is massless and the background solution will be BPS in the sense of the solution generating technique utilized in ref. 13. In general, the $\sigma_{i}$ depend on parameters of the brane potentials, and they determine the zero-mode masses for the radion and the bulk scalar field in terms of the curvature scale $k$. The effects of various choices of $\sigma_{i}$ will be explored in section 4 .

\subsection{The remaining Einstein equations}

So far we have succeeded in finding the back-reaction as a function of the radion fluctuation $\varphi$, but we have not yet determined the dynamics of $\varphi$. This comes from solving the remaining Einstein equations. They are difficult to solve exactly due to their explicit dependence on $y$ through the warp factor $n(y)$ which also appears in the metric and scalar perturbations. However, when we supplement the restrictions (3.18) on $f_{2}$ and $g_{2}$ by analogous ones for $f, g$,

$$
f, g \ll \varphi \quad \text { and } \quad f, g \ll 1
$$

then it is possible to linearize to obtain some equations that have negligible $y$-dependence. The radion will induce only a small back-reaction on the $4 \mathrm{D}$ slices of the $5 \mathrm{D}$ spacetime relative to the static solution,

$$
d s_{4}^{2} \simeq e^{2 n(y)}\left(d t^{2}-a^{2} d \mathbf{x}^{2}\right)\left(1+2 n f(\varphi)+2 f_{2}(\varphi) e^{-2\left(n-n_{1}\right)}+\cdots\right)
$$

Similar to the off-diagonal equation (3.15), zeroth order terms vanish since these involve factoring out $\varphi$ dependence with the background solutions. The remaining equations can then be averaged over the extra dimension to find the radion dynamics:

$$
\int_{0}^{1} d y \sqrt{g} G_{N}^{M}=\kappa_{5}^{2} \int_{0}^{1} d y \sqrt{g} T_{N}^{M}
$$

It was shown in reference [23] that the cosmology of the radion in the RS model can be obtained equivalently from the effective action or from averaging the Einstein equations as in (3.37). In Section 4.1 we will construct the $4 \mathrm{D}$ effective action that leads to the bulk-averaged equations of motion.

The bulk equations to be averaged are given in the Appendix, eqs. (A.2)-(A.4). Collecting $t$ derivatives to the right hand side, Using eq. (2.7) for $V$ and (3.12, 3.14) for $\Phi$, linearizing 
eq. (3.37) with $\kappa_{5}^{2}=3 \mu_{5}^{-2}$ and integrating over $y$ we find to first order in the back-reaction functions

$$
\begin{array}{r}
\left(\frac{\dot{a}}{a}\right)^{2}+\frac{\dot{a}}{a} \dot{\varphi}\left(1+\Upsilon_{1}\right)-\dot{\varphi}^{2}\left(\frac{1}{2}+\Upsilon_{2}\right)=3 k^{2} e^{-2 \varphi}\left(f-g-\Upsilon_{5}\right) \\
2 \frac{\ddot{a}}{a}+\left(\frac{\dot{a}}{a}\right)^{2}+\left(2 \frac{\dot{a}}{a} \dot{\varphi}+\ddot{\varphi}\right)\left(1+\Upsilon_{1}\right)+\dot{\varphi}^{2}\left(\frac{5}{2}+\Upsilon_{3}\right)=9 k^{2} e^{-2 \varphi}\left(f-g-\Upsilon_{5}\right) \\
\frac{\ddot{a}}{a}+\left(\frac{\dot{a}}{a}\right)^{2}+\ddot{\varphi}\left(\frac{\Upsilon_{1}}{2}+\Upsilon_{4}^{\prime}\right)+3 \frac{\dot{a}}{a} \dot{\varphi}\left(\frac{\Upsilon_{1}}{2}+\Upsilon_{4}\right)+\frac{\dot{\varphi}^{2}}{2}\left(1+\frac{\Upsilon_{2}+\Upsilon_{3}}{2}+2 \Upsilon_{4}\right) \\
=k^{2} e^{-2 \varphi}\left(4 f-3 g-\Upsilon_{6}\right)
\end{array}
$$

where primes denote $\frac{d}{d \varphi}$ and

$$
\begin{array}{r}
\Upsilon_{1} \equiv-\frac{2}{3} f^{\prime}\left(1-3 k b_{0} e^{-3 k b_{0}} \Omega\right), \quad \Upsilon_{2} \equiv-\frac{1}{2} \Upsilon_{1}-g^{\prime}-3 e^{-3 k b_{0}}\left(f_{2}^{\prime}+g_{2}^{\prime}\right)\left(1-e^{k b_{0}}\right) \Omega \\
\Upsilon_{3} \equiv-\Upsilon_{2}-4 g^{\prime}-12 e^{-3 k b_{0}}\left(f_{2}^{\prime}+g_{2}^{\prime}\right)\left(1-e^{k b_{0}}\right) \Omega, \quad \Upsilon_{4} \equiv 3 e^{-3 k b_{0}} f_{2}^{\prime}\left(1-e^{k b_{0}}\right) \Omega \\
\Upsilon_{5} \equiv g_{2} e^{-3 k b_{0}}\left(1-e^{k b_{0}}\right) \Omega, \quad \Upsilon_{6} \equiv 3 e^{-3 k b_{0}}\left(2 f_{2}+5 g_{2}\right)\left(1-e^{k b_{0}}\right) \Omega
\end{array}
$$

with

$$
\Omega=\left(1-e^{-3 k b_{0}}\right)^{-1}
$$

All of the $\Upsilon_{i}$ terms are subdominant to other similar terms appearing in the equations, so it is consistent to drop them and keep only the explicit $f$ and $g$ source terms (notice that $\Upsilon_{5,6}$ are exponentially suppressed by powers of the warp factor). This demonstrates our earlier assertion that the radion dynamics are dominated by the Planck brane potential, which determines $f$ and $g$, while $f_{2}$ and $g_{2}$ are needed for stabilization only. The remaining Einstein equations then take the simple FRW-like form

$$
\begin{aligned}
\left(\frac{\dot{a}}{a}\right)^{2}+\frac{\dot{a}}{a} \dot{\varphi}-\frac{\dot{\varphi}^{2}}{2} & =3 k^{2} e^{-2 \varphi}(f-g) \\
2 \frac{\ddot{a}}{a}+\left(\frac{\dot{a}}{a}\right)^{2}+\ddot{\varphi}+2 \frac{\dot{a}}{a} \dot{\varphi}+\frac{5}{2} \dot{\varphi}^{2} & =9 k^{2} e^{-2 \varphi}(f-g) \\
\frac{\ddot{a}}{a}+\left(\frac{\dot{a}}{a}\right)^{2}+\frac{\dot{\varphi}^{2}}{2} & =k^{2} e^{-2 \varphi}(4 f-3 g)
\end{aligned}
$$

These equations are augmented by the linearized Klein-Gordon equation

$$
\ddot{\varphi}+3 \frac{\dot{a}}{a} \dot{\varphi}+\dot{\varphi}^{2}=4 k^{2} e^{-2 \varphi}\left(f-\frac{3}{2} g\right)
$$

found at this level of approximation by integrating (A.1) or equivalently by starting from the $4 \mathrm{D}$ effective action (4.3) in Jordan frame which we derive in the next section.

Of course, not all four of the equations (3.45-3.48) are independent. As was observed in ref. [22], the linear combination of equations $(3.48)-(3.46)+2 \times(3.47)$ is equivalent to eq. (3.45). Furthermore, $\frac{d}{d t}(3.45)+H(3 \times(3.45)-(3.46)) / \dot{\varphi}+(3.48)+(3.45)$ is equivalent to eq. (3.47). The two constraints arise as a consequence of the gauge symmetries, namely reparametrizations of the $t$ and $y$ coordinates [22], 223]. 


\section{Inflation in the effective $4 \mathrm{D}$ theory}

It is not difficult to derive the form of the effective action that gives rise to the $4 \mathrm{D}$ equations

of motion (3.45)-(3.48). Starting from the 5D action (2.1) and the metric (2.2) one arrives at

$$
\begin{array}{r}
S=2 \int_{0}^{1} d^{5} x \sqrt{g}\left[\mu_{5}^{2}\left(-e^{-2 N}\left(\dot{A}^{2}+\dot{A} \dot{B}\right)+2 e^{-2 B} N^{\prime 2}\right)+\frac{1}{2} \partial^{\mu} \Phi \partial_{\mu} \Phi-V(\Phi)\right] \\
-\left.\int d^{4} x \sqrt{g_{4}} V_{0}\right|_{y=0}-\left.\int d^{4} x \sqrt{g_{4}} V_{1}\right|_{y=1}
\end{array}
$$

The boundary contributions from the compact extra dimension exactly cancel the extrinsic curvature terms and so do not appear in this expression. Substituting for the metric functions $A, B$ and $N$ in (3.10), for $V$ and $\Phi$ with (2.7) and (3.12), and using the jump condition (3.19) to rewrite $V_{i}$ in terms of the perturbations one can linearize about $\Phi=\mu_{5}\left(k b_{0} y-\varphi\right)$. After integrating over $y$ we arrive at the effective action

$$
S=\frac{2 \mu_{5}^{2}}{3 k \Omega} \int a^{3} e^{\varphi} d t\left[-\left(\frac{\dot{a}}{a}\right)^{2}-\frac{\dot{a}}{a} \dot{\varphi}\left(1+\Upsilon_{1}\right)+\dot{\varphi}^{2}\left(\frac{1}{2}+\Upsilon_{2}\right)-3 k^{2} e^{-2 \varphi}\left(f-g-\Upsilon_{5}\right)\right]
$$

Neglecting the small $\Upsilon_{i}$ terms as before, we can write the simplified effective action in Jordan frame as

$$
S=\frac{1}{2 \kappa_{4}^{2}} \int d t a^{3} e^{\varphi}\left(-6\left(\frac{\dot{a}}{a}+\frac{1}{2} \dot{\varphi}\right)^{2}+\frac{9}{2} \dot{\varphi}^{2}-2 \kappa_{4}^{2} V_{r, J}(\varphi)\right)
$$

where $V_{r, J}=9 k^{2} \kappa_{4}^{-2} e^{-2 \varphi}(f-g)$ and the $4 \mathrm{D}$ Newton's constant $\kappa_{4}^{2}=8 \pi G$ is found from

$$
\kappa_{4}^{-2}=2 \kappa_{5}^{-2} \int_{0}^{1} b_{0} e^{-3 k b_{0} y} d y=\frac{2 \mu_{5}^{2}}{9 k \Omega}
$$

It is straightforward to show that this action implies the equations of motion (3.45)-(3.48) to linear order in $f, g$ when we make use of eq. (3.16).

One can go to the Einstein frame by performing the Weyl transformation

$$
a(t)=\gamma \bar{a}(\tau), \quad d t=\gamma d \tau, \quad \gamma=e^{-\frac{\varphi}{2}}
$$

after which the action takes the form

$$
S=\frac{1}{2 \kappa_{4}^{2}} \int d \tau \bar{a}^{3}\left(-6\left(\frac{\dot{\bar{a}}}{\bar{a}}\right)^{2}+\frac{9}{2} \dot{\varphi}^{2}-2 \kappa_{4}^{2} V_{r}(\varphi)\right)
$$

where dots now denote $\frac{d}{d \tau}$, and the radion no longer mixes with the scale factor. The Einstein frame potential is

$$
V_{r}=\frac{9 k^{2}}{\kappa_{4}^{2}} e^{-3 \varphi}(f-g)
$$

and $\varphi$ is related to the canonically normalized radion field $\phi$ by

$$
\phi=\frac{3}{\sqrt{2}} \kappa_{4}^{-1} \varphi=\frac{3}{\sqrt{2}} m_{p} \varphi \equiv \mu_{4} \varphi
$$


where $m_{p}$ is the reduced Planck mass. The Friedmann equation then takes the usual form

$$
\bar{H}^{2}=\frac{\kappa_{4}^{2}}{3}\left(\frac{1}{2} \dot{\phi}^{2}+V_{r}\right)
$$

as does the Klein-Gordon equation

$$
\ddot{\phi}+3 \bar{H} \dot{\phi}=-\frac{d V_{r}}{d \phi}
$$

We will continue to do some of the subsequent analysis using the dimensionless field $\varphi$ however, to avoid having to repeatedly write $\mu_{4}$.

\subsection{Radion stability}

To summarize the results to this point, we have shown that in the class of $5 \mathrm{D}$ warped models we are considering, the radion potential is given by

$$
V_{r}(\phi)=9 k^{2} m_{p}^{2} e^{-3 \phi / \mu_{4}}(f-g)
$$

where the functions $f$ and $g$ are determined by the Planck brane potential $V_{0}$ to leading order for small $f, g$, by eqs. (3.26, 3.27). The TeV brane potential $V_{1}$ has virtually no effect because of the suppression of $f_{2}$ and $g_{2}$ by powers of the warp factor in eqs. (3.21. 3.22 ).

We are assuming that $\phi=0$ is a stable equilibrium point. Since $f=g=f^{\prime}-g^{\prime}=0$ at $\phi=0$ (see eq. (3.16)), it is clear that $\phi=0$ is a critical point of the potential, and $V_{r}$ also vanishes at this point, leading to a Minkowski solution. To compute the radion mass, notice that $f^{\prime \prime}-g^{\prime \prime}=f^{\prime}$. Therefore

$$
m_{r}^{2}=\left.\frac{d^{2} \bar{V}_{r}}{d \phi^{2}}\right|_{\phi=0}=\frac{9 k^{2} m_{p}^{2}}{\mu_{4}^{2}} f^{\prime}(0)=2 k^{2} f^{\prime}(0)=2 k^{2} g^{\prime}(0)
$$

Using eq. (3.21), we find that $g^{\prime}(0)=1-2 k / V_{0}^{\prime \prime}(0)$. (Recall that $g^{\prime}=\frac{d g}{d \varphi}$ and $V_{0}^{\prime \prime}=\frac{d^{2} V_{0}}{d \Phi^{2}}$.)

Consider for example the exponential potentials (2.16-2.17). The radion mass is determined by $V_{0}^{\prime \prime}(0)=2 k\left[\alpha_{0}^{2}\left(1-\beta_{0}\right)-\beta_{0}^{2}\left(1-\alpha_{0}\right)\right] /\left(\alpha_{0}-\beta_{0}\right)$. Recalling our discussion of the hierarchy problem, where it was pointed out that values of $\alpha_{1}$ and $\beta_{1}$ close to unity alleviate the need for a strong explicit hierarchy between the scales of the potentials on the two branes, we are motivated to define

$$
\alpha_{1}=1+\hat{\alpha}, \quad \beta_{1}=1+\hat{\beta}
$$

and for consistency when comparing parameters

$$
\alpha_{0}=1+\alpha, \quad \beta_{0}=1+\beta
$$

Then it is straightforward to show that $g^{\prime}(0)=\alpha \beta(1+\alpha \beta) /(1-\alpha \beta)$. The radion mass squared is thus given by

$$
m_{r}^{2}=-2 k^{2} \alpha \beta \frac{1+\alpha \beta}{1-\alpha \beta}
$$


and there is a second zero mode whose mass has a similar expression in terms of the $\hat{\alpha}$ and $\hat{\beta}$ parameters. Hence $m_{r}^{2}$ is generically nonzero, and it is positive as long as $-1<\alpha \beta<0$ or $\alpha \beta>1$. As for the remaining parameters we will show in the next subsection that $0<\hat{\alpha} \hat{\beta} \ll 1$ is required in order to avoid a tachyon instability in the second light state, and to address the hierarchy problem.

We emphasize an important difference between our model and the RS model supplemented by Goldberger-Wise stabilization of the radion. In the latter, the radion mass is suppressed by the warp factor and so is naturally at the TeV scale, whereas in ours, whose background geometry is different, it is natually at the scale $k$; we must do one tuning of parameters, $\alpha \ll 1$, to make it small enough for inflation. This will be advantageous when we consider the robustness of the radion-as-inflaton potential against quantum corrections from standard model physics later on.

\subsection{Kaluza-Klein excitations}

One might wonder if it is sufficient to stabilize the radion without also considering the zero mode of the bulk scalar field. As ref. 21] has shown, there is only a single KK tower for the two fields. However, in our model there is a second zero mode in the tower, the sign of whose mass squared depends on the choice of brane potentials. We will show that this state can be made much heavier than the radion so that it is consistent to neglect it along with the higher KK modes. Our main point is to show that the nonzero KK modes are not excited during inflation, which if it happened would invalidate our effective $4 \mathrm{D}$ description. We will find that these modes have masses of order $k$, while the second zero mode hass mass $\sqrt{\sigma_{1}} k$, with $\sigma_{i}$ defined in (3.34). The details of the calculations are left to Appendix B.

Following Csaki, Graesser and Kribs (CGK) [21] we consider the spectrum of perturbations around the background solution. The metric ansatz used to describe the scalar fluctuations differs slightly from theirs since we are working with a $y$-coordinate in which the background is conformally flat

$$
\begin{aligned}
d s^{2} & =e^{2 n(y)}\left[e^{2 F(x, y)} \eta_{\mu \nu} d x^{\mu} d x^{\nu}-e^{2 G(x, y)} d y^{2}\right] \\
\Phi(x, y) & =\Phi_{b}(y)+\delta \Phi(x, y)=\mu_{5} k b y+\delta \Phi(x, y)
\end{aligned}
$$

A similar analysis was subsequently done by Kofman, Martin and Peloso (KMP) [24]. They generalized the CGK results by constructing a gauge invariant combination of scalar perturbations which ensures the hermiticity and orthogonality of modes in the mass spectrum, defined here as

$$
v \equiv z\left(F+\frac{\delta \Phi}{\mu_{5}}\right)
$$

with

$$
z \equiv \sqrt{2} \frac{\Phi_{b}^{\prime}}{n^{\prime}} e^{\frac{3 n}{2}}=-\sqrt{2} \mu_{5} e^{-\frac{3 k y}{2}}
$$

$v$ is analogous to the Mukhanov-Sasaki variable [25, 26] in 4D inflationary cosmology. Expanding the action (2.1) to second order and diagonalizing in terms of the different scalars, 
one finds separable solutions $v(x, y)=\sum_{j} Q_{j}(x) \tilde{v}_{j}(y)$ such that the quadratic action of the KK modes can be reduced to [24]

$$
S=\sum_{j} C_{j} \int d^{4} x Q_{j}\left[-\square-m_{j}^{2}\right] Q_{j}
$$

with the normalization coefficients

$$
\begin{aligned}
C_{j} & \equiv \frac{1}{2} \int_{0}^{1} d y b_{0} \tilde{v}_{j}^{2}+\left.\frac{\mu_{5}^{2}}{k} e^{3 n} \tilde{F}_{j}^{2}\right|_{y_{0}=0} ^{y_{1}=1} \\
& =\frac{1}{2} \int_{0}^{1} d y b_{0} e^{3 n}\left(\frac{1}{k b_{0}} \tilde{F}_{j}^{\prime}-3 \tilde{F}_{j}\right)^{2}+\left.\frac{\mu_{5}^{2}}{k} e^{3 n} \tilde{F}_{j}^{2}\right|_{y_{0}=0} ^{y_{1}=1}
\end{aligned}
$$

where $\tilde{F}_{j}$ is related to $\tilde{v}_{j}$ as $F$ is to $v$ in 4.18). The solutions are determined by the jump conditions (B.6, B.7) and the two nondynamical constraints (B.3, B.4 ) originating from the linearized off-diagonal Einstein equations to determine $G$ and $\delta \Phi$.

To calculate the mass spectrum in our model we incorporate the results of KMP and CGK except in the application of the boundary conditions. Unlike the present work, refs. [21], [24] assumed a convenient stiff potential limit for the stabilizing potentials that forces $\delta \Phi$ to vanish on the branes with the result that only the radion zero mode appears with the infinite tower of KK excitations. Relaxing this assumption gives rise to the bulk scalar zero mode. One can also understand the two light modes as being the moduli of the positions of the two branes in the bulk [27].

General solutions obtained from the action expanded at second order for $v_{j}$ are equivalent to those obtained for $F_{j}$ from the linearized Einstein equations [24]. We prefer to solve for $F_{j}$ similarly to CGK since the boundary conditions and normalization look simpler in this language. The heavier KK excitations and the zero modes take the form

$$
\begin{aligned}
& \tilde{F}_{j}=e^{-\frac{3}{2} k b_{0} y}\left(A_{j} \sin \left(\sqrt{\lambda}_{j} b_{0} y\right)+B_{j} \cos \left(\sqrt{\lambda}_{j} b_{0} y\right)\right) \\
& \tilde{F}_{z}=e^{-\frac{3}{2} k b_{0} y}\left(A_{z} e^{\sqrt{\lambda_{z}} b_{0} y}+B_{z} e^{-\sqrt{\lambda} b_{0} y}\right)
\end{aligned}
$$

where

$$
\lambda_{j}=m_{j}^{2}-\frac{9 k^{2}}{4} \quad, \quad \lambda_{z}=\frac{9 k^{2}}{4}-m_{z}^{2}
$$

for $4 m_{j}^{2} \geq 9 k^{2}$ and $m_{z}^{2} \ll k^{2}$ respectively. The index $j \geq 0$ is an integer which labels the heavier KK modes, while $z=r, s$ stands for the two light (radion or bulk scalar) zeromodes. The complete solutions are obtained by applying the linearized boundary conditions to determine the ratio of integration constants and the mass.

The lightest KK excited state has $\lambda_{0}=0$ with $m_{0}^{2}=\frac{9}{4} k^{2}$. Heavier modes must be

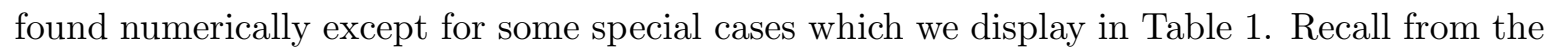
discussion in the previous subsection that $\sigma_{1}=\hat{\alpha} \hat{\beta} \ll 1$ is needed to explain the hierarchy between the Planck and $\mathrm{TeV}$ branes. If $\left|\sigma_{0}\right| \ll\left|\sigma_{1}\right| \ll 1$ so as to achieve a large mass gap 


\begin{tabular}{l|l|l}
\multicolumn{1}{c|}{$\sigma_{i}$} & $m_{j}^{2}$ (KK modes) & $m_{z}^{2}$ (zero modes) \\
\hline$\sigma_{0}<0, \sigma_{1}>0$, & $m_{0}^{2}=\frac{9}{4} k^{2}$ & $m_{r, s}^{2} \simeq-2 \sigma_{0} k^{2}, \sigma_{1} k^{2}$ \\
$\left|\sigma_{0}\right| \ll\left|\sigma_{1}\right| \ll 1$ & $m_{j+1}^{2} \simeq \frac{9}{4} k^{2}+\left[\left(j+\frac{1}{2}\right) \frac{\pi}{b_{0}}\right]^{2}$ & \\
\hline$\sigma_{i}=0$ & $\frac{9}{4} k^{2}+\left(\frac{j \pi}{b_{0}}\right)^{2}$ & 0 \\
$\left|\sigma_{i}\right| \gg 1$ & $\frac{9}{4} k^{2}+\left(\frac{j \pi}{b_{0}}\right)^{2}$ & $2 k^{2}$ \\
$\sigma_{0}=\sigma_{1},\left|\sigma_{i}\right| \ll 1$ & $\frac{9}{4} k^{2}+\left(\frac{j \pi}{b_{0}}\right)^{2}$ & $m_{r, s}^{2} \simeq-2 \sigma_{0} k^{2}, \sigma_{0} k^{2}$
\end{tabular}

Table 1: A summary of the mass spectrum for various brane potentials parametrized by $\sigma_{i}$. The entries correspond to the general case where tachyons are absent, followed by the massless radion, the stiff potential limit and fine-tuned brane potentials.

between the radion and the other modes, then the KK masses are well-approximated by

$$
m_{j+1}^{2} \cong \frac{9}{4} k^{2}+k^{2}\left[\left(j+\frac{1}{2}\right) \frac{\pi}{k b_{0}}\right]^{2}
$$

for $j \geq 0$. Thus all KK mode masses are at least order $k$, independent of the precise details of the stabilizing potentials.

We previously showed that the radion mass depends on $V_{0}$, the potential on the Planck brane; see eq. (4.12)). Similarly the bulk scalar zero mode mass depends on the TeV brane potential $V_{1}$. In the case where the brane potentials are tuned such that $\left|\sigma_{i}\right| \ll 1$ (eq. (3.34)) the zero mode masses are approximately

$$
m_{r}^{2} \cong-2 \sigma_{0} k^{2}, \sigma_{1} k^{2}
$$

To avoid tachyonic instabilities we must impose $\sigma_{0}<0$ and $\sigma_{1}>0$. This result agrees with the analysis of criteria regarding the presence of tachyons for brane world models utilizing the Goldberger-Wise mechanism carried out in reference [28].

Comparing (4.26) with (4.15), for the exponential brane potential $V_{0}$ (2.16) which implies $\sigma_{0}=\alpha \beta$, we corroborate at first order in $\sigma_{0}$ the more exact expression for the radion mass given in the previous subsection. We will henceforth assume that $\sigma_{1}=\hat{\alpha} \hat{\beta}$ from $V_{1}$ (2.17) is large enough so that the bulk scalar zero mode is much heavier than the radion:

$$
\left|\sigma_{0}\right| \ll \sigma_{1} \ll 1
$$

We will verify that $m_{s} \gg H$ (the Hubble scale during inflation) so that it is consistent to ignore the bulk scalar zero mode during inflation. The KK modes are much heavier and thus can also be considered as frozen. 


\subsection{Radion inflation: an explicit model}

Let us now consider the full radion potential (valid to leading order in $f, g$ ) corresponding to the brane potentials (2.16 2.17), with the restrictions (2.18 2.19) and the definitions (4.14). Ignoring the small exponential terms involving $f_{2}$ and $g_{2}$ we find that

$$
g=\frac{(\alpha-\beta) e^{\beta \varphi}+\beta(1+\alpha) e^{(\beta-\alpha) \varphi}-\alpha(1+\beta)}{\alpha(1+\beta)^{2}-\beta(1+\alpha)^{2} e^{(\beta-\alpha) \varphi}}
$$

and the combination $(f-g)$ that enters the radion potential (4.11) is

$$
\begin{aligned}
f-g & =\frac{1+g}{\alpha-\beta}\left[\beta-\alpha+\alpha(1+\beta) e^{-\beta \varphi}-\beta(1+\alpha) e^{-\alpha \varphi}\right] \\
& +g \frac{\alpha \beta}{\alpha-\beta}\left(e^{-\beta \varphi}-e^{-\alpha \varphi}\right)
\end{aligned}
$$

In the case we are interested in where $|\hat{\alpha}|,|\hat{\beta}| \ll 1$ in (4.13), the expressions for $f_{2}$ and $g_{2}$ (3.28, 3.29) take the simple form then

$$
\begin{aligned}
& f_{2} \simeq-\frac{1}{2} f+g+\frac{1}{2} \hat{\alpha} \hat{\beta}\left(\varphi-\varphi^{2}\right) \\
& g_{2} \simeq f-g+\frac{1}{2} \hat{\alpha} \hat{\beta} \varphi^{2}
\end{aligned}
$$

Since $\varphi \sim 1$ during inflation while $\hat{\alpha} \hat{\beta} \ll 1$, it follows that if $f$ and $g$ are small then so are $f_{2}$ and $g_{2}$ and the consistency conditions (3.18) are satisfied.

To design a suitable potential for inflation, we have the two dimensionless parameters $\alpha, \beta$ and one dimensionful scale $k$ at our disposal, subject to the restriction (4.27) on $\sigma_{0}=\alpha \beta$. In addition $\sigma_{1}=\hat{\alpha} \hat{\beta}$ must satisfy (4.27) and we must ensure that $f, g \ll 1$ for consistency of the effective theory. Notice from (4.28) that if $|\alpha| \sim|\beta|$, then generically $g \sim 1$ even if $|\alpha|,|\beta| \ll 1$. However if

$$
|\alpha| \ll|\beta|
$$

(or $\beta \ll \alpha$, giving qualitatively similar results) then $f$ and $g$ can be made parametrically small: both vanish in the limit $\alpha \rightarrow 0$. This motivates our choice of parameter values. We have also remarked that the radion potential has a second degenerate (Minkowski) minimum because of the decompactification limit as $\varphi \rightarrow \infty$. Thus the generic form of the potential is that of a hilltop model with a barrier separating the minima at $\varphi=0$ and $\varphi=\infty$. We find that this barrier can be made very flat when $\beta$ is close to -3 , for $0<\alpha \ll 1$, making it possible to get inflation from near the top of the barrier. The shape of the potential is illustrated for $\alpha=10^{-7}$ and a few values of $\beta$ near -3 in figure 1. Figure 2 shows that the condition $f, g \ll 1$ is satisfied in the region $0<\varphi<3$ where inflation starting from the top of the barrier might take place. Choosing $\hat{\alpha}=10^{-2}$ and $\hat{\beta}=6 \times 10^{-3}$ for the fiducial values of $\alpha=10^{-7}$ and $\beta=-3$ is sufficient to ensure eqs. (4.30,4.31) for $f_{2}$ and $g_{2}$ are good approximations and that the mass squared of the second light zero mode is much larger 


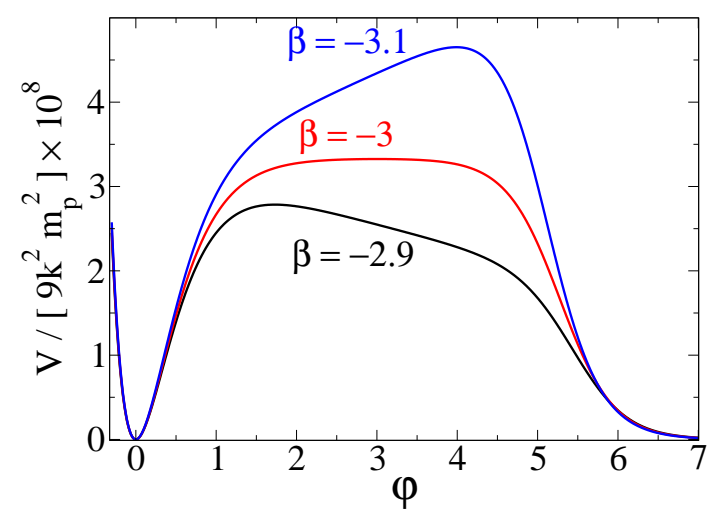

Figure 1: Radion potential for $\alpha=10^{-7}$ and $\beta=-2.9,-3-3.1$.

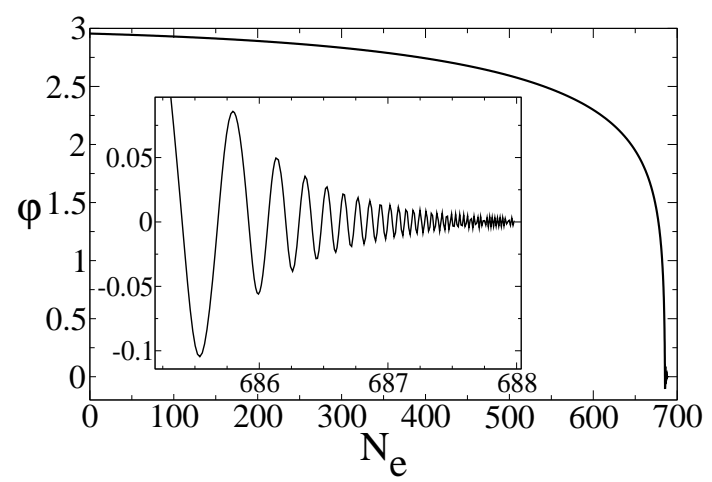

Figure 3: $\varphi$ as a function of number of $e$ foldings $N$ for $\alpha=10^{-7}, \beta=-3$ potential, starting $\Delta \varphi=0.01$ from the top of the barrier. Inset shows end of inflation.

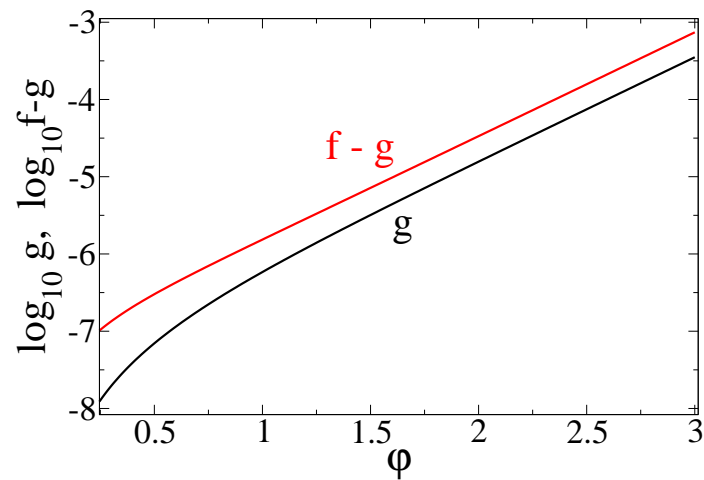

Figure 2: Logarithm of the functions $g$ and $f-g$ that must be $\ll 1$ in the region of validity of the potential, for the case $\alpha=10^{-7}$ and $\beta=-3$.

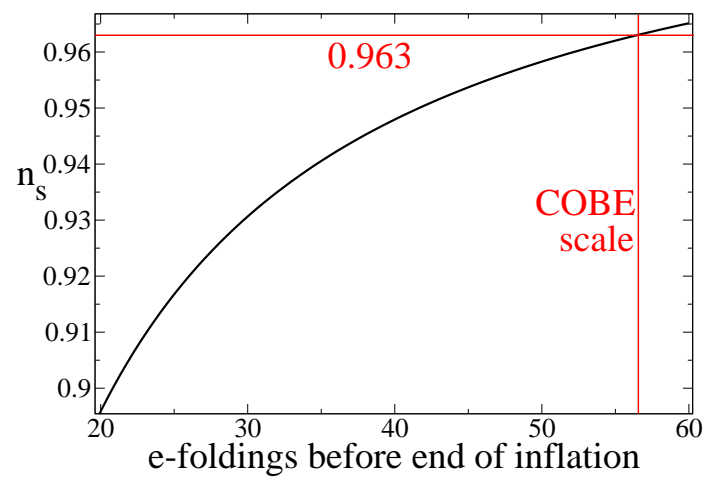

Figure 4: Spectral index as a function of number of $e$-foldings until the end of inflation, corresponding to fig. 3 .

than that of the radion. We will presently show that it also exceeds the Hubble scale during inflation.

We have numerically integrated the inflaton equations of motion starting close to the top of the barrier. Taking the number of $e$-foldings $N$ as the time variable, these can be written in the first-order form,

$$
\frac{d \varphi}{d N}=\frac{\pi}{\mu_{4} H} ; \quad \frac{d \pi}{d N}=-3 \pi-\frac{1}{\mu_{4} H} \frac{d V_{r}}{d \varphi}
$$

where $\pi=\dot{\varphi}$ is the canonical momentum and the Hubble parameter is given by $H^{2}=\frac{1}{6} \pi^{2}+\frac{1}{3} V_{r}$ in Planck units. (Recall that $\mu_{4}$ arises in the relation between the canonically normalized radion $\phi$ and the dimensionless one $\varphi$.) For $\alpha=10^{-7}, \beta=-3$, the maximum of the potential is at $\varphi_{m}=3.01$. Starting at $\varphi=\varphi_{m}-\delta \varphi$ with $\delta \varphi=0.05$, for example, gives $N_{e}=685$ $e$-foldings of inflation. $\varphi$ as a function of $N$ is shown in figure 3 . Although the total number 
of $e$-foldings depends upon how small $\delta \varphi$ is taken to be, the spectral index at the time of horizon crossing (nominally $60 e$-foldings before the end of inflation) is insensitive to the initial condition, as long as at least $60 e$-foldings of inflation occurred. This behavior has been observed in a number of previous studies of hilltop inflation models [29]-[34].

To compute the power spectrum and spectral index we have used the prescription (see eq. (43) of ref. [35])

$$
\mathcal{P}=\frac{1}{150 \pi^{2}} \frac{V_{r}}{m_{p}^{4} \epsilon}, \quad n_{s}=1+\frac{d \ln \mathcal{P}}{d N}
$$

in terms of the slow roll parameter $\epsilon=\frac{1}{2} m_{p}^{2}\left(V_{r}^{\prime} / V_{r}\right)^{2}=\frac{1}{9} m_{p}^{2}\left(\frac{d V_{r}}{d \varphi} / V_{r}\right)^{2}$. These are to be evaluated at horizon crossing, approximately $60 e$-foldings before the end of inflation. More precisely, we take the crossing of the scale relevant for the COBE normalization of the power spectrum to be $N_{\mathrm{COBE}}=56.5$ [31], a number that depends only logarithmically on the scale of inflation; since the spectrum changes slowly with $N$, this is an adequate approximation for our purposes.

Fig. 1 shows the spectral index $n_{s}$ as a function of $N_{e}-N$, the number of $e$-foldings until the end of inflation. We see that $n_{s}=0.96$ at the scale of horizon crossing, in agreement with the WMAP5 central value. The normalization of the power spectrum at this point, $\sqrt{\mathcal{P}}=2 \times 10^{-5}$, implies that the curvature scale is given by $k=0.015 m_{p}$. Eq. (4.15) shows that the radion mass is

$$
m_{r} \cong \sqrt{6 \alpha} k=1.2 \times 10^{-5} m_{p}=3 \times 10^{13} \mathrm{GeV} .
$$

From fig. 1 the scale of inflation $m_{I}=V^{1 / 4}$ is given by $m_{I}^{4} \cong 3 \times 10^{-8} \cdot 9 k^{2} m_{p}^{2}$ in the flat region of the potential, hence

$$
m_{I}=2.7 \times 10^{-3} m_{p}
$$

The first slow roll parameter is $\epsilon=1.1 \times 10^{-4}$, giving a small tensor-to-scalar ratio of $r=1.8 \times 10^{-3}$. This is related to the fact that the canonically normalized inflaton field does not change by an amount much greater than the Planck scale [36]; in our model $\Delta \phi=\frac{3}{\sqrt{2}} m_{p} \Delta \varphi \cong 6 m_{p}$. This is consistent with a refined version of the Lyth bound [37], $\Delta \phi \cong 6 M_{p} r^{1 / 4}\left(\right.$ where $\left.M_{p}=\sqrt{8 \pi} m_{p}\right)$.

We investigated the effect of varying the parameters $\alpha$ and $\beta$ away from the fiducial values $\alpha=10^{-7}$ and $\beta=-3$. Holding $\beta$ fixed and varying $\alpha$, we find that for small $\alpha$ the spectral index asymptotes to its maximum value of $n_{s}=0.963$ (figure 5). Figure 6 shows that the curvature scale $k$, determined by the normalization of the power spectrum, follows a power-law relation $k \sim \alpha^{-1 / 2}$ for very small values of $\alpha$. Recall that the prefactor of the inflaton potential is proportional to $k^{2} m_{p}^{2}$; however the actual scale of inflation does not rise despite the increase in $k$; the $\epsilon$ slow roll parameter asymptotes to $1.2 \times 10^{-4}$ at the smallest values of $\alpha$, which is nearly the same as its value at $\alpha=10^{-7}$.

The corresponding results of varying $\beta$ at fixed $\alpha=10^{-7}$ are shown in figures 7 and 8 . We see that it is possible to achieve a higher value of the spectral index than by varying only $\alpha$; the maximum value is $n_{s}=0.99$ at $\beta=-3.1$. 


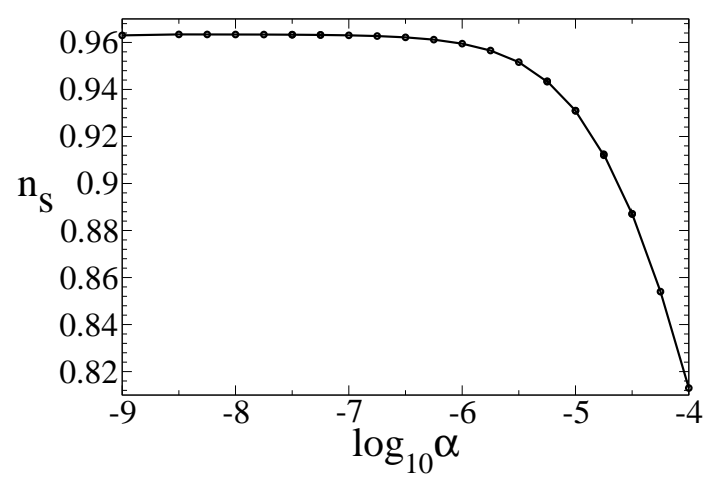

Figure 5: Spectral index versus $\log _{10} \alpha$ for $\beta=-3$ model.

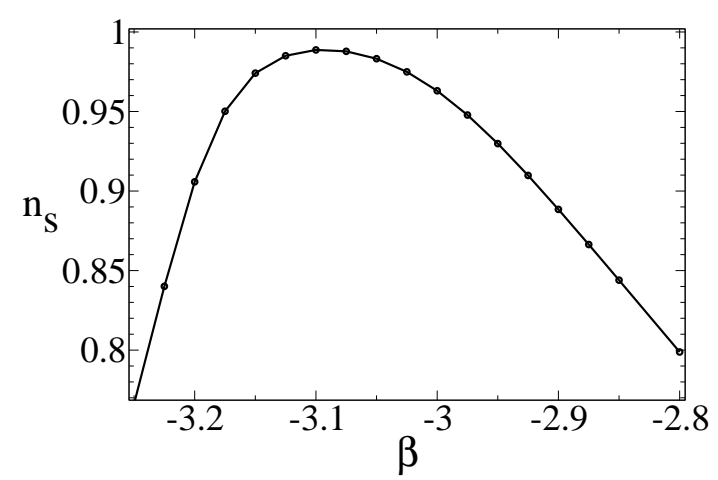

Figure 7: Spectral index versus $\beta$ for $\alpha=$ $10^{-7}$ model.

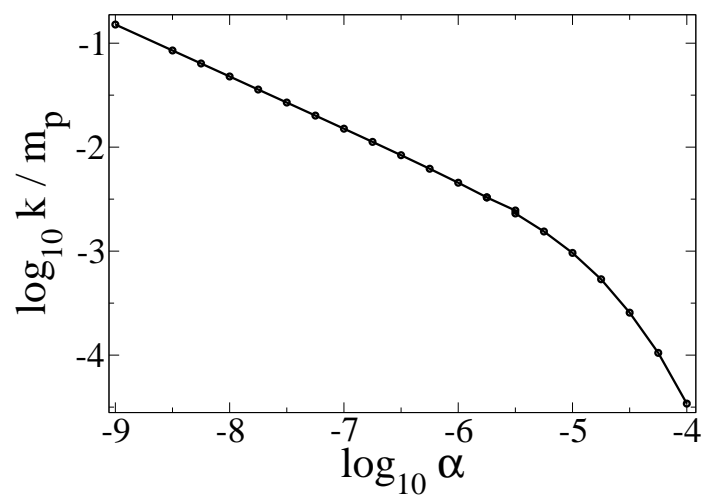

Figure 6: $\log$ of curvature scale $k$ versus $\log _{10} \alpha$ for $\beta=-3$ model.

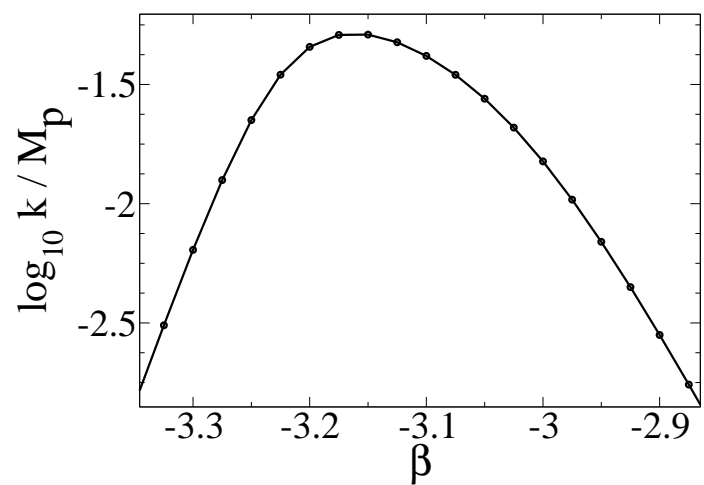

Figure 8: $\log$ of curvature scale $k$ versus $\beta$ for $\alpha=10^{-7}$ model.

\subsection{Model with larger tensors}

In the previous model, constructed from brane potentials consisting of just two exponentials, there was not enough freedom to find examples with a tensor-to-scalar ratio $r$ exceeding $10^{-3}$. However with the addition of just one more exponential term, we can overcome this limitation. Consider the Planck brane potential

$$
V_{0}=\Lambda_{0} e^{\alpha_{0} \Phi / \mu_{5}}-\Delta_{0} e^{\beta_{0} \Phi / \mu_{5}}-\Omega_{0} e^{\delta_{0} \Phi / \mu_{5}}
$$

The back-reaction functions $f$ and $g$ are given by

$$
\begin{aligned}
& f=-1+e^{\varphi}\left(\Lambda_{0} e^{-\alpha_{0} \varphi}-\delta_{0} e^{-\beta_{0} \varphi}+\Omega_{0} e^{-\delta_{0} \varphi}+g\left(\Lambda_{0} \alpha_{0} e^{-\alpha_{0} \varphi}-\delta_{0} \beta_{0} e^{-\beta_{0} \varphi}+\Omega_{0} \delta_{0} e^{-\delta_{0} \varphi}\right)\right) \\
& g=-\frac{e^{-\varphi}-\Lambda_{0} \alpha_{0} e^{-\alpha_{0} \varphi}+\Delta_{0} \beta_{0} e^{-\beta_{0} \varphi}-\Omega_{0} \Delta_{0} e^{-\Delta_{0} \varphi}}{-\Lambda_{0} \alpha_{0}{ }^{2} e^{-\alpha_{0} \varphi}+\Delta_{0} \beta_{0}{ }^{2} e^{-\beta_{0} \varphi}-\Omega_{0} \Delta_{0}{ }^{2} e^{-\Delta_{0} \varphi}}
\end{aligned}
$$

which lead to the radion potential (4.11), proportional to $e^{-3 \varphi}(f-g)$. 
Solving the unperturbed junction conditions similarly to eq. (2.18 2.19), we obtain

$$
\begin{aligned}
& \frac{\Lambda_{0}}{2 \mu_{5}^{2} k}=\frac{1}{\beta-\alpha}\left(\beta+\frac{\Omega_{0}}{2 \mu_{5}^{2} k}(\beta-\delta)\right) \\
& \frac{\Delta_{0}}{2 \mu_{5}^{2} k}=\frac{1}{\beta-\alpha}\left(\alpha+\frac{\Omega_{0}}{2 \mu_{5}^{2} k}(\alpha-\delta)\right)
\end{aligned}
$$

where we have reparametrized $\delta_{0}=\delta+1$ similarly to (4.14). This model therefore has two parameters in addition to the previous one, $\delta$ and $\Omega_{0}$, the latter of which we find convenient to exchange for $\Omega_{0} / 2 \mu_{5}^{2} k \equiv h \alpha$. It will turn out that interesting values of $\Omega_{0} / 2 \mu_{5}^{2} k$ are of order $\alpha$, so that $h$ is of order unity.

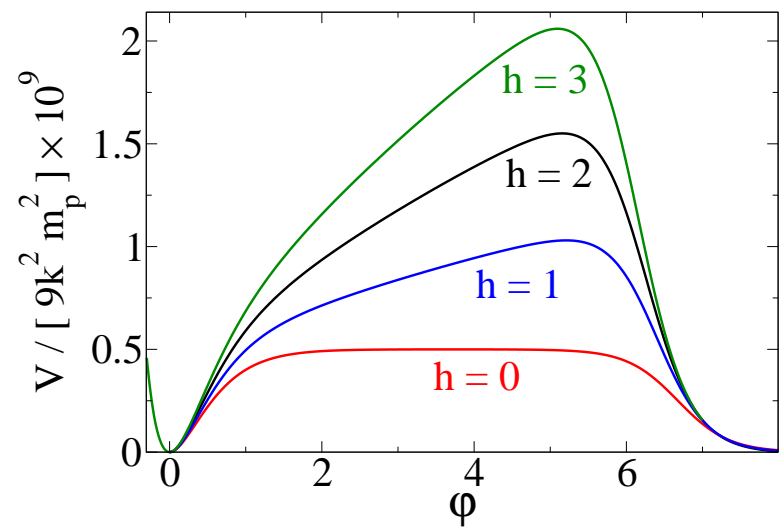

Figure 9: Potential of extended model for $h=$ $0,1,2$, and $\alpha=1.5 \times 10^{-9}, \beta=-3, \delta=-2.9$.

this possibility.

First consider the small- $\alpha$ approximation to the potential, which can be written as

$$
V_{r} \cong 9 k^{2} m_{P}^{2} \alpha\left[\frac{1-\delta h-3 h e^{-(\delta+3) \varphi}-e^{-3 \varphi}(1+3 \varphi-h(\delta+3))}{3+\alpha e^{3 \varphi}\left(4(1-\delta h)-3 h(1+\delta)^{2} e^{-(\delta+3) \varphi}\right)}\right]
$$

This is a good approximation in the inflationary region, between $\varphi=0$ and the maximum of the potential. To see the turnover of the potential at large $\varphi$, it is important to keep the term of $O(\alpha)$ in the denominator; neglecting it leads to a potential that levels out at large $\varphi$ instead of having a maximum. Next consider the case where $0<\delta+3 \ll 1$ so that the terms of order $e^{-3 \varphi}$ in the numerator are subdominant. This leads to

$$
V_{r} \cong 9 k^{2} m_{P}^{2} \alpha\left[\frac{1-\delta h-3 h e^{-(\delta+3) \varphi}}{3+\alpha V_{\delta h}(\varphi)}\right]
$$

The term $\alpha V_{\delta h}(\varphi)$ is $O(1)$ near the maximum of the potential, but it becomes subdominant away from the maximum, and the $\varphi$-dependence is dominated by the numerator. In this

\footnotetext{
${ }^{3}$ See ref. 38] for another recent example leading to a linear potential, or ref. [39] for other string-derived potentials supposed to be valid at Planckian field values.
} 
region, since $|\delta+3| \ll 1$, the dependence is in fact linear. We can write $V_{r} \sim(1-\xi+3 \xi \varphi)$, where $\xi=h(3+\delta)$. This potential has slow-roll parameters $\eta \cong 0$ and

$$
\epsilon=\frac{1}{2}\left(\frac{m_{p}^{2}}{\mu_{4}^{2}}\right)\left(\frac{V_{r}^{\prime}}{V}\right)^{2}=\frac{\xi^{2}}{(1-\xi+3 \xi \varphi)^{2}}
$$

Inflation ends when $\epsilon \sim 1$, i.e.,

$$
\varphi_{e}=\frac{2 \xi-1}{3 \xi}
$$

It is straightforward to solve the slow-roll equation of motion for the linear potential. Setting $d \pi / d N=0$ in eq. (4.33), we have $d \varphi / d N=-\left(m_{p} / \mu_{4}\right)^{2} V_{r}^{\prime} / V_{r}$. This gives a simple quadratic function for $N(\varphi)$. Using (4.43), one can find $\varphi$ as a function the number of $e$-foldings before the end of inflation,

$$
\varphi(N)=\frac{1}{3}\left(\sqrt{1+4 N}+1-\xi^{-1}\right)
$$

Notice that with $N \cong 60$ and $\xi \cong 1, \varphi(60) \cong 5$, in agreement with the position of the maximum of the potential in fig. 9. Substituting this into (4.42) we get a simple result in which the dependence on the slope parameter $\xi$ drops out completely:

$$
\epsilon=\frac{1}{4 N+1} \cong 0.0044
$$

where we have taken $N=56.5$ to correspond to the COBE scale. This gives a tensor-to-scalar ratio of

$$
r=16 \epsilon=0.07
$$

which is somewhat higher than the projected sensitivity of the Planck experiment, $r_{\min } \cong 0.05$ [40]. Moreover the spectral index is

$$
n_{s}=1-6 \epsilon=0.974
$$

The prediction that $r$ is independent of the parameters of the potential in this regime depends on the assumption that the linear behavior in the numerator of (4.41) dominates over the $\varphi$-dependence from $V_{\delta h}(\varphi)$ in the denominator. In the next subsection we will quantify this by solving the equations of motion numerically using the full potential.

Using eqs. (4.44 4.45), the power spectrum (4.34) in this model is $P=3\left(150 \pi^{2}\right)^{-1} \alpha \xi k^{2} m_{p}^{2}$ $(4 N+1)^{3 / 2}$, allowing us to determine $\sqrt{\alpha} k$ through the normalization:

$$
\sqrt{\alpha} k=7.6 \xi^{-1 / 2} \times 10^{-6} m_{p}
$$

The radion mass differs from that in (4.35) only by an extra factor of $\sqrt{1+\xi} \sim 1$. 


\subsubsection{Numerical analysis}

Numerical analysis shows that the linear behavior of the potential is fully achieved for somewhat smaller values of $\alpha \lesssim 10^{-11}$ than we considered previously. For larger values of $\alpha$, the linear shape is not fully realized and the tensor ratio does not reach its maximum value of $r=0.07$. This is illustrated in figures 10 and 11, which show the dependence of $r$ and $n_{s}$ on $h$ and $\delta$ respectively, when $\alpha=1.5 \times 10^{-9}$. On the other hand, by decreasing $\alpha$ to $1.5 \times 10^{-12}$ and tuning $\delta$ closer to -3 , the predictions (4.46) and (4.47) based on the linear potential are borne out, both for $r$ and for $n_{s}$. In all cases, values of $h(3+\delta)$ of order unity are needed to get the maximum tensor signal.

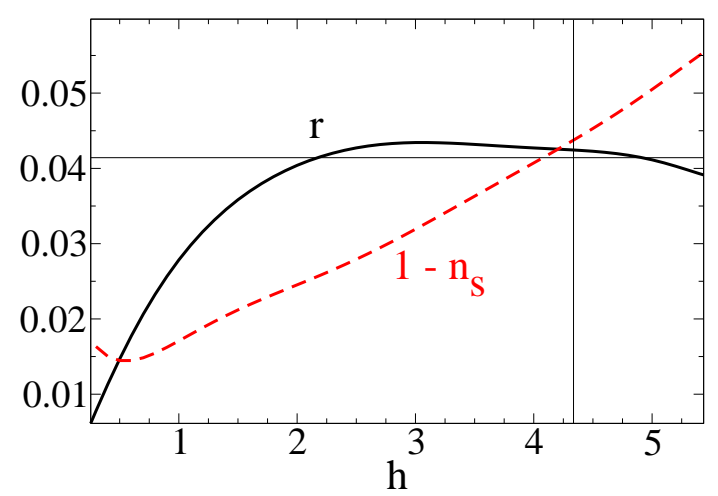

Figure 10: Tensor ratio and $1-n_{s}$ versus $h$ for $\alpha=1.5 \times 10^{-9}, \beta=-3, \delta=-2.9$.

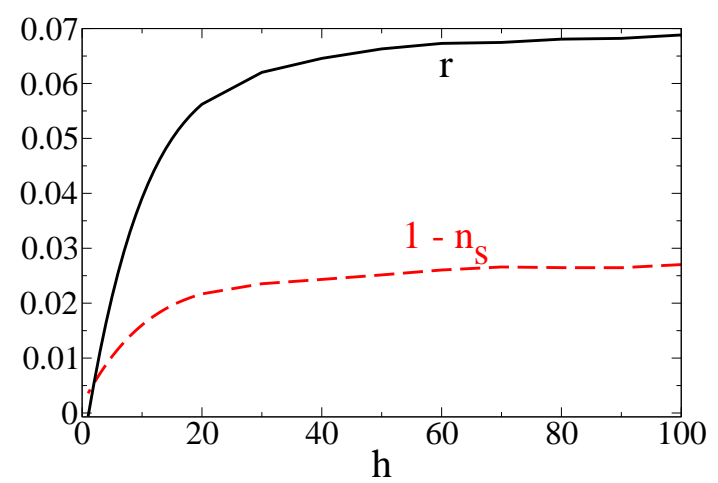

Figure 12: Same as fig. 10, but for $\alpha=1.5 \times$ $10^{-12}$ and $\delta=-2.99$.

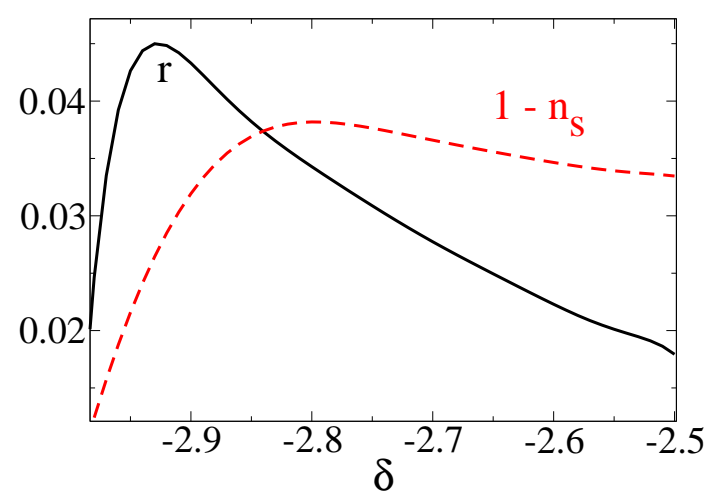

Figure 11: Tensor ratio and $1-n_{s}$ versus $\delta$ for $\alpha=1.5 \times 10^{-9}, \beta=-3, h=3$

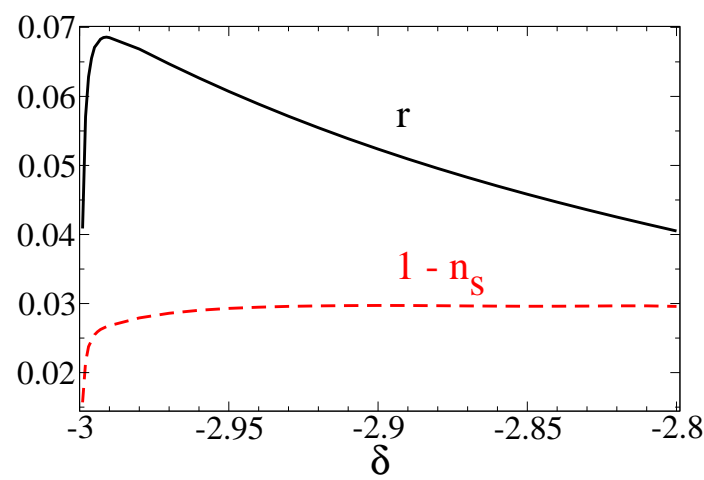

Figure 13: Same as fig. 11, but for $\alpha=1.5 \times$ $10^{-12}$ and $h=100$.

It is encouraging that the model is able to produce values of $r$ that exceed the minimum value of $r \cong 0.05$, which is estimated to be at the threshold for detection by the Planck satellite [40]. It is novel that we find such a regime in a hilltop potential [30], for which the tensor ratio is typically unobservably small, $r<0.002$. We have checked that for the examples shown here, the maximum value of $f, g$ in the inflationary region is $f=0.02$, so the 
condition $f, g \ll 1$ is still satisfied and the $O\left(f^{2}, g^{2}\right)$ corrections to our approximation for the potential are under control.

Consistent with the analytic prediction (4.48), the curvature scale $k$ starts to exceed $m_{p}$ in the large-tensor models that have the smallest values of $\alpha$. For example with $1.5 \times 10^{-11}$, $\delta=-2.99$ and $h=200$, we obtain $r=0.066$ and $k=1.4 m_{p}$. Examples with nearly as large tensors exist with $k<m_{p}$, such as $\alpha=5 \times 10^{-11}, \delta=-2.985, h=100$, yielding $r=0.06$ and $k=0.8 m_{p}$. However it is the combination $\sqrt{\alpha} k$ that appears in the potential, and this remains subPlanckian, so the fact that $k$ itself may exceed the Planck scale can be regarded as merely an artifact of our parametrization.

\section{Coupling of radion to standard model}

An interesting feature of this model that distinguishes it from most other models of inflation is that the couplings of the inflaton to the standard model are exactly specified, since the radion is a component of the higher-dimensional metric. This enables us to address the details of reheating in a way that is usually not possible without making additional assumptions. Moreover, we can compute the quantum corrections to the radion potential to check whether its exotic form is radiatively stable.

The most natural situation is that the SM fields are localized on the $\mathrm{TeV}$ brane. As an example, consider a Higgs field $H$. Using the metric (3.10) and the conformal transformation (4.5), we find that in the Einstein frame, its coupling to $\varphi$ is

$$
\mathcal{L}_{\varphi, H}=e^{-\varphi-2 k b_{0} f+2 f_{2}}(\partial H)^{2}-e^{-2 \varphi-4 k b_{0} f+4 f_{2}} m_{H}^{2} H^{2}
$$

where we have renormalized the field and the mass to absorb warp factors so that $m_{H}$ is the already-warped mass, $m_{H} \lesssim \mathrm{TeV}$. For small $\varphi, f(\varphi) \sim \alpha \varphi$ and $f_{2}(\varphi) \sim \hat{\alpha} \hat{\beta} \varphi$ which in the inflationary models we have considered is a negligible correction to the leading $\varphi$ dependence in the exponents, giving the usual result that the radion couples to the trace of the stressenergy tensor.

\subsection{Reheating}

Using the conventional theory of perturbative reheating, one estimates the reheat temperature as $T_{r} \sim g_{*}^{-1 / 4}\left(\Gamma M_{p}\right)^{1 / 2}$ where $\Gamma$ is the decay rate of the inflaton and $g_{*}$ the number of relativistic degrees of freedom. Using the interaction (5.1) and recalling the relation (4.8) between $\varphi$ and the canonically normalized radion $\phi$ gives a rate of order $\Gamma \sim g_{*} m_{H}^{4} /\left(16 \pi m_{p}^{2} m_{r}\right)$, since there are $\sim g_{*}$ additional degrees of freedom besides the Higgs into which the radion can decay. For $m_{H} \sim 100 \mathrm{GeV}$, this leads to a reheat temperature that is too low even for nucleosynthesis, $T_{r} \sim 10^{-3} \mathrm{eV}$.

However, there exists a much more efficient channel for reheating, by decay of the radion into gauge bosons. Although the radion does not couple to gauge bosons at tree level due to the tracelessness of their stress energy tensor, the conformal anomaly induces a coupling at 
one loop, of the form [21]

$$
\frac{\alpha}{8 \pi} \varphi F_{\mu \nu} F^{\mu \nu}
$$

where $\alpha$ is the fine structure constant. An analogous term is present for gluons and the electroweak vector bosons. The decay rate due to this operator is much faster than that due to (5.1) since the derivatives are of order $m_{r} / 2$ as opposed to $m_{H} \cdot{ }^{4}$ The decay rate is approximately

$$
\Gamma \sim \frac{\alpha^{2} m_{r}^{3}}{3^{2} 2^{13} \pi^{3} m_{p}^{2}}
$$

Using the radion mass (4.35) and $\alpha=0.1$ for $\mathrm{QCD}$, this gives a reheat temperature of order

$$
T_{r} \sim 10^{7} \mathrm{GeV}
$$

which is high enough for electroweak baryogenesis, and low enough to avoid the gravitino problem.

It is possible that reheating could be more efficient than indicated by this perturbative estimate, due to parametric resonance by the same coupling. Production of massless particles can be a particularly efficient form of preheating (see for example ref. [41]).

\subsection{Radiative corrections to inflaton potential}

The radion potentials we have derived have exotic shapes compared to simple renormalizable potentials, and this has enabled us to produce distinctive signatures, like a larger tensor ratio than would be expected. One should always be concerned whether such peculiar potential shapes are radiatively stable. A second advantage of knowing the radion's couplings to matter is that we can address this question quantitatively. Consider the coupling (5.1) to a Higgs field. To compute the contribution to the effective potential for $\varphi$, we can take $\varphi$ to be constant and renormalize $H$ to have a canonical kinetic term. Then the contribution to the Coleman-Weinberg potential is

$$
\Delta V \sim e^{-2 \varphi} \frac{m_{H}^{4}}{64 \pi^{2}}
$$

Since $m_{H}^{4} \ll k^{2} m_{p}^{2}$ (the prefactor of the radion potential), this is a negligible correction. Of course the more relevant contributions come from integrating out particles at some intermediate scale $m_{\text {int }}$, if there is new physics above the $\mathrm{TeV}$ scale. Even then however we are safe as long as $m_{\text {int }}^{4} \ll k^{2} m_{p}^{2}$.

One correction that does not appear in the Coleman-Weinberg potential is the cosmological constant on the branes, a constant shift to the brane potentials (2.16-2.17). Such terms can be studied in the extended model with brane potential (4.37) in the limit where $\delta_{0}=0$ and by adding a similar term to the TeV brane. We have checked that our conclusions based of the potential with two exponentials in 4.3 are not very sensitive to the addition of the constant term. Fig. 14 shows the effect of the constant term on the potential: for $h>0$, the flat

\footnotetext{
${ }^{4}$ we thank Neil Barnaby for pointing out the possibility of perturbative decay into gauge bosons
} 
region of the hilltop is shortened, leading to a shorter period of inflation, while for $h<-1$ it is destabilized. The spectral index as a function of $h$ is shown for the same potentials in fig. 15; values up to $h \cong 20$ are compatible with the $1 \sigma$ WMAP5 allowed region, $n_{s}=0.963 \pm 0.015$.

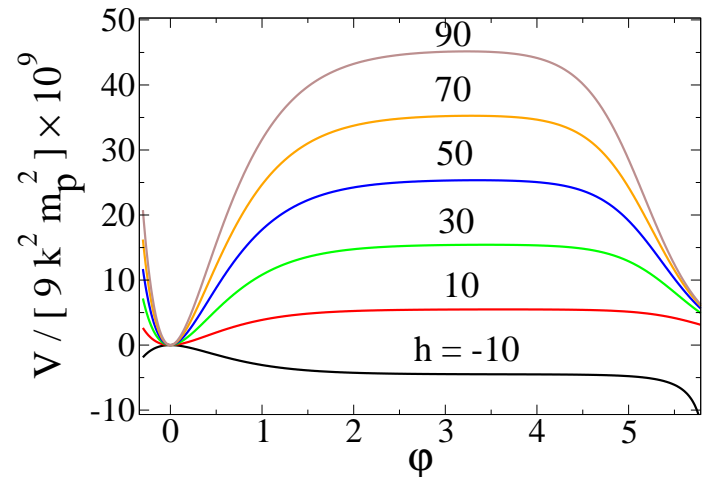

Figure 14: Effect of constant addition to brane potential on inflaton potential, parametrized as eq. (4.17) with $\Omega_{0} / 2 \mu_{5}^{2} k \equiv$ $h \alpha$, for $\alpha=1.5 \times 10^{-9}, \beta=-3$, and a range of values of $h=-10,10, \ldots, 90$.

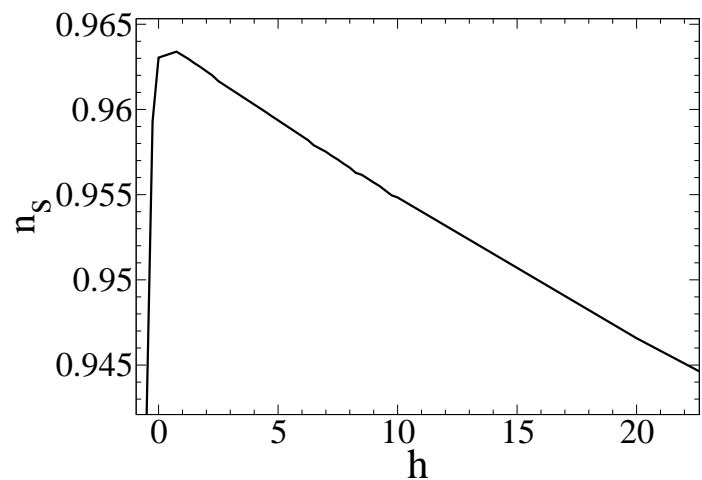

Figure 15: Spectral index as a function of $h$ for the potentials of fig. 14 .

\section{Discussion}

We have explored a new framework for inflation, where the radion in a warped 5D compactification is the inflaton. Our approach differs from the usual Randall-Sundrum setup in that we work in the linear dilaton background (a bulk scalar that varies linearly with the extra dimension) rather than $\mathrm{AdS}_{5}$. The bulk scalar stabilizes the radion by the Goldberger-Wise mechanism. Knowing its unperturbed solution exactly gives us greater computational control over the back-reaction when we perturb the radion away from the minimum of its potential. We derived an approximate analytic formula for the radion potential that depends mainly on the form of bulk scalar potential term on the Planck brane.

Similar to the RS model, the hierarchy between the weak and Planck scales arises in our model without having to build it into the 5D Lagrangian. One can check that for the dimensionless parameter values we needed for inflation, the dimensionful brane potential parameters (2.18, 2.19) are dominated by the terms $\Lambda_{0}$ and $\Delta_{1}$, both of order $\mu_{5}^{2} k$ for a warp factor consistent with $k b_{0} \sim 37$ needed to solve the hierarchy problem. On the other hand, the mass of the radion does not get warped down to the weak scale; $m_{r}$ is fixed to be around $10^{13} \mathrm{GeV}$ by the normalization of the CMB power spectrum. Moreover the couplings of the radion are not enhanced by the inverse warp factor; they remain Planck-suppressed. This tends to lead to a low reheat temperature, but also protects the inflaton potential from large radiative corrections.

Choosing a sum of two exponentials as the brane potentials, we found that the radion potential suitable for inflation was generically of the hilltop form, but (with some tuning of 
dimensionless couplings) having a shape that could be much flatter than the generic negative quadratic form. Expanding the ansatz on the Planck brane to a sum of three exponential enabled us to find potentials where the descent from the hilltop is linear and consistent with a large tensor contribution $r=0.07$, detectable by Planck.

An advantage of this model is that the couplings of the radion to matter on the branes can be computed explicitly. The radion couples to the trace of the stress energy tensor of a given particle, and to the one-loop conformal anomaly for states that are classically conformally invariant. We showed that this leads to reheating into gauge bosons by perturbative decay, with a reheat temperature of order $T_{r} \sim 10^{7} \mathrm{GeV}$. We did not explore the possibility of parametric resonance, which might significantly increase $T_{r}$.

A direction for further development is to see if our scenario can be realized in a string theory compactification. It is well-known that warped-throat-like linear dilaton solutions arise in the near horizon region of a stack of NS 5-branes [42] (see also section 14.1 of [43]). Moreover, in the same way as the Klebanov-Strassler throats [44 are smoothly capped versions of anti-de Sitter throats near a stack of D3-branes, there exist known smoothly capped throatlike linear dilaton solutions [45], [46]. So far these throats and their compactifications have not been as widely studied as the analogous Klebanov-Strassler throat.

In ref. 47] it was proposed that dynamically weakening gravity at early times could provide the low-entropy initial conditions that are needed for inflation to get started. In the Jordan frame our effective 4D action (4.3) has a time-dependent Newton's constant $\hat{G}(\phi)=$ $G e^{-\phi / \mu_{4}}$ when the radion is displaced from its equilibrium value, which could potentially realize this scenario. In this frame, $4 \mathrm{D}$ gravity is effectively weak when the radion is far from its stable minimum, which is also the condition needed for inflation. During inflation $\hat{G}(\phi)$ grows to its normal strength and then oscillates about this value as the radion decays. It would be interesting to further investigate the extent to which our model is compatible with this proposal.

Another feature of our model is that the mass spectrum of general scalar perturbations, including the zero modes of the radion and bulk scalar field and the infinite tower of KK excitations, is completely known in terms of $k$ and the parameters of the brane potentials. All modes can be normalized and their exact 4D effective actions determined. This presents an opportunity for constructing a model of assisted inflation [48] which we will explore elsewhere [49].

Acknowledgements: We thank Andrew Frey, Alex Maloney and Johannes Walcher for information about the linear dilaton background, Neil Barnaby for insights about reheating and preheating, and Cliff Burgess for discussions about radiative corrections to the brane potentials. 


\section{A. Bulk field equations}

The ansatz of eq. (2.2) leads to the scalar field equation

$$
\frac{d}{d t}\left(e^{-N+3 A+B} \dot{\Phi}\right)-\frac{d}{d y}\left(e^{N+3 A-B} \Phi^{\prime}\right)+e^{N+3 A+B}\left[\frac{d V}{d \Phi}+\frac{d V_{0}}{d \Phi} \delta(y)+\frac{d V_{1}}{d \Phi} \delta(y-1)\right]
$$

and after substituting into the Einstein equations, $G_{M N}=\kappa^{2} T_{M N}$ we have

$$
\begin{aligned}
G_{0}^{0} & =3 e^{-2 N}\left(\dot{A}^{2}+\dot{A} \dot{B}\right)-3 e^{-2 B}\left(A^{\prime \prime}+2 A^{\prime 2}-A^{\prime} B^{\prime}\right) \\
G_{i}^{i} & =e^{-2 N}\left(2 \ddot{A}+3 \dot{A}^{2}+2 \dot{A} \dot{B}-2 \dot{A} \dot{N}-\dot{N} \dot{B}+\ddot{B}+\dot{B}^{2}\right) \\
& -e^{-2 B}\left(2 A^{\prime \prime}+3 A^{\prime 2}+N^{\prime \prime}+N^{\prime 2}+2 A^{\prime} N^{\prime}-2 A^{\prime} B^{\prime}-N^{\prime} B^{\prime}\right) \\
G_{5}^{5} & =3 e^{-2 N}\left(\ddot{A}+2 \dot{A}^{2}-\dot{A} \dot{N}\right)-3 e^{-2 B}\left(A^{\prime 2}+A^{\prime} N^{\prime}\right) \\
G_{05} & =3\left(N^{\prime} \dot{A}+A^{\prime} \dot{B}-A^{\prime} \dot{A}-\dot{A}^{\prime}\right)
\end{aligned}
$$

in the bulk where the indices are $M, N=0,1,2,3,5$. The bulk contribution to the stress energy tensor comes from

$$
T_{M N}=g_{M N} V+\partial_{M} \Phi \partial_{N} \Phi-\frac{1}{2}\left(\partial^{l} \Phi \partial_{l} \Phi\right) g_{M N}
$$

and we have the sources on the Planck and TeV branes:

$$
\begin{aligned}
T_{N}^{M}= & e^{-B(0, t)} \delta(y) \operatorname{diag}\left(V_{0}, V_{0}, V_{0}, V_{0}, 0\right) \\
& +e^{-B(1, t)} \delta(y-1) \operatorname{diag}\left(V_{1}, V_{1}, V_{1}, V_{1}, 0\right)
\end{aligned}
$$

We do not consider the effects of adding matter to the branes, since we are primarily interested in the cosmology generated by the dynamical evolution of the radion coupled to the bulk.

\section{B. Kaluza-Klein excitations}

In this Appendix we provide details necessary for the computation of the KK mass spectrum summarized in Section 4.2. We closely follow the methods of CGK [21] and KMP [24] and highlight differences in the zero mode solutions resulting from our different choice of stabilizing potentials on the branes. In particular, we have a second light mode due to not taking the stiff potential limit.

Starting from the action of eq. (2.1) we expand to second order in the scalar fluctuations (4.16) to arrive at 24]

$$
\begin{aligned}
S= & \frac{1}{2} \int d^{5} x v\left[-\square+\frac{d^{2}}{d y^{2}}-\frac{z^{\prime \prime}}{z}\right] v \\
& +\int d^{5} x \partial_{y}\left[\frac{\mu_{5}^{2} e^{3 n}}{n^{\prime}} \eta^{\mu \nu} \partial_{\mu} F \partial_{\nu} F+\frac{e^{3 n}}{n^{\prime}} F\left(4 n^{\prime} \Phi_{b}^{\prime}-e^{2 n} V^{\prime} \pm \frac{e^{n} V_{i}^{\prime \prime} \Phi_{b}^{\prime}}{2}\right) \delta \Phi\right]
\end{aligned}
$$


where we define $v$ and $z$ as in eqs. (4.18,4.19). The eigenvalue equations for separable solutions to $v=\sum_{j} Q_{j}(x) \tilde{v}_{j}(y)$ are in Schrödinger form

$$
\left[-\square+\frac{d^{2}}{d y^{2}}-\frac{z^{\prime \prime}}{z}\right] v_{j}=0
$$

while the second term of (B.1) is not yet in a simplified form. To reach the compact form given by (4.20) in 24] we use an equivalent set of eigenvalue equations involving $F=\sum_{j} Q_{j}(x) \tilde{F}_{j}(y)$ which can be derived from the linearized Einstein equations.

There are two nondynamical equations that we use to fix $G$ and $\delta \Phi$ in terms of $F$. The first comes from recognizing that $\partial_{\mu} \partial_{\nu}$ terms at linear order should vanish [21] because there are no off-diagonal contributions in the stress energy tensor to source such terms. $\delta G_{\mu \nu}=0$ for these $4 \mathrm{D}$ components gives

$$
G=-2 F
$$

which has already been applied in (B.1). The second constraint comes from the 5D linearized off-diagonal Einstein equation, $\delta G_{\mu 5}=\kappa_{5}^{2} \delta T_{\mu 5}$. Inserting (B.3) it can be integrated to find

$$
\delta \Phi=-\frac{\mu_{5}^{2}}{\Phi_{b}^{\prime}}\left(F^{\prime}+2 n^{\prime} F\right)
$$

where a $y$-dependent "constant" of integration has been set to zero to ensure localization of the perturbations in $x$ (the large dimensions). With these constraints, the system of linearized Einstein equations is then reduced to a single dynamical equation in the bulk found from the combination $\delta G_{\mu}^{\mu}-2 \delta G_{5}^{5}$

$$
F^{\prime \prime}+3 n^{\prime} F^{\prime}=\square F
$$

where one also eliminates $V$ (eq. (2.7)) using the background solutions and the property $d V / d \Phi=2 \mu_{5}^{-1} V$ of our bulk potential. Applying the definition (4.18) and the constraint (B.4) for eigenmodes $\square F_{j}=-m_{j}^{2} F_{j}$, eq. (B.5) is seen to be equivalent to that derived from the action involving $v_{j}$ (eq. (B.2)).

To determine the mass spectrum we must apply the linearized boundary conditions. At the Planck $(+)$ and $\mathrm{TeV}(-)$ branes,

$$
\begin{aligned}
\left.b_{0}^{-1} e^{-n} F^{\prime}\right|_{y_{i}-\epsilon} ^{y_{i}+\epsilon} & = \pm\left.\left. 2 F \mu_{5}^{-2} V_{i}\left(\Phi_{i}\right)\right|_{y_{i}} \mp \delta \Phi \mu_{5}^{-2} V_{i}^{\prime}\left(\Phi_{i}\right)\right|_{y_{i}} \\
\left.b_{0}^{-1} e^{-n} \delta \Phi^{\prime}\right|_{y_{i}-\epsilon} ^{y_{i}+\epsilon} & =\left.\mp 2 F V_{i}^{\prime}\left(\Phi_{i}\right)\right|_{y_{i}} \pm\left.\delta \Phi V_{i}^{\prime \prime}\left(\Phi_{i}\right)\right|_{y_{i}}
\end{aligned}
$$

where primes on the potentials denote $\frac{d}{d \Phi}$. The ansatz for small fluctuations (4.16) makes (B.4) redundant with the first boundary condition. The second can be simplified using eq. (3.34) where we recall that the two would-be zero-modes get squared masses proportional to $\sigma_{1}$ and $\sigma_{2}$. If we also rewrite eq. (B.5) in Schrödinger-like form

$$
\mathcal{F}_{j}^{\prime \prime}-\frac{9}{4} n^{\prime 2} \mathcal{F}_{j}=-m_{j}^{2} \mathcal{F}_{j}
$$


by defining

$$
F \equiv e^{-3 n / 2} \mathcal{F}
$$

so that

$$
\left(\square+m_{j}^{2}\right) Q_{j}=0, \quad\left(\frac{d^{2}}{d y^{2}}-\frac{9}{4} n^{\prime 2}+m_{j}^{2}\right) \tilde{\mathcal{F}}_{j}=0
$$

and if we moreover apply eqs. (B.4, B.5) at the boundaries, then (B.7) reduces to

$$
\left.\tilde{\mathcal{F}}_{j}^{\prime}\right|_{y=y_{i}}=\left.\kappa_{i j} k b_{0} \tilde{\mathcal{F}}_{j}\right|_{y=y_{i}}
$$

We have defined

$$
\kappa_{i j} \equiv \frac{m_{j}^{2}}{\sigma_{i} k^{2}}+\frac{1}{2}
$$

such that the index $i$ identifies the brane position $\left(y=y_{i}\right)$ and $j$ is an integer corresponding to the KK mode which we set to $z=r, s$ when treating the light radion and bulk scalar modes.

Solutions to $(\mathrm{B} .8)$, which we provide below, and consequently also to $(\overline{\mathrm{B} .2})$ when inserted back into the second order action (B.1), reduce to the effective actions of the KK modes given by eq. (4.20). We reproduce them here with the normalization coefficients given in terms of $\tilde{\mathcal{F}}_{j}$ :

$$
\begin{aligned}
S & =\sum_{j} C_{j} \int d^{4} x Q_{j}\left[-\square-m_{j}^{2}\right] Q_{j} \\
C_{j} & \equiv \mu_{5}^{2} \int_{0}^{1} d y b_{0}\left(\frac{\tilde{\mathcal{F}}_{j}^{\prime}}{k b_{0}}-\frac{3 \tilde{\mathcal{F}}_{j}}{2}\right)^{2}+\left.\frac{\tilde{\mathcal{F}}_{j}^{2}}{k}\right|_{y_{0}=0} ^{y_{1}=1}
\end{aligned}
$$

We have also substituted for the bulk potential (2.7), eliminated the $V_{i}^{\prime \prime}$ terms in favour of perturbations using the boundary conditions (B.7), and applied the constraint equation (B.4) for $\delta \Phi$.

The general solutions to the eigenvalue equations $(\bar{B} .10)$ can be written similarly to eqs. $(4.22,4.23)$ for $\tilde{F}_{j}$

$$
\begin{aligned}
& \tilde{\mathcal{F}}_{j}=A_{j} \sin \left(\sqrt{\lambda}_{j} y\right)+B_{j} \cos \left(\sqrt{\lambda}_{j} y\right) \\
& \tilde{\mathcal{F}}_{z}=A_{z} e^{\sqrt{\lambda_{z}} y}+B_{z} e^{-\sqrt{\lambda}_{z} y}
\end{aligned}
$$

where as before

$$
\lambda_{j}=m_{j}^{2}-\frac{9 k^{2}}{4} \quad, \quad \lambda_{z}=\frac{9 k^{2}}{4}-m_{z}^{2}
$$

for $4 m_{j}^{2} \geq 9 k^{2}$ and $m_{z}^{2} \ll k^{2}$ respectively.

Solving first for the KK modes, at $y=y_{0}=0$ the constants are related by

$$
A_{j}=-\frac{B_{j} k \kappa_{0 j}}{\sqrt{\lambda_{j}}}
$$


At $y=y_{1}=1$ we get a transcendental equation which is solved to find the masses

$$
k \sqrt{\lambda_{j}}\left(\kappa_{1, j}-\kappa_{0 j}\right) \cos \left(\sqrt{\lambda_{j}} b\right)=\left(k^{2} \kappa_{1 j}-\lambda_{j}\right) \sin \left(\sqrt{\lambda_{j}} b\right)
$$

The lightest KK excitation solution always gives $\lambda_{0}=0$ with $m_{0}^{2}=\frac{9}{4} k^{2}$. The heavier modes must be found numerically except for the special cases of the stiff potential limit $\left(\left|\sigma_{i}\right| \gg 1\right)$, a massless radion $\left(\sigma_{i}=0\right)$, fine-tuned brane potentials $\left(\sigma_{0}=\sigma_{1}\right)$ or that of our model where a large mass gap exists between the radion zero mode and the rest of the tower. In the former cases one solves $\sin \left(\sqrt{\lambda_{j}} b\right)=0$ to determine the masses. In our model when $\left|\sigma_{0}\right| \ll\left|\sigma_{1}\right| \ll 1$ we find $\cos \left(\sqrt{\lambda_{j}} b\right) \simeq 0$ and

$$
m_{j+1}^{2} \simeq \frac{9}{4} k^{2}+k^{2}\left[\left(j+\frac{1}{2}\right) \frac{\pi}{k b_{0}}\right]^{2}
$$

We next turn our attention to the zero-mode solutions. Following the same reasoning using eq. (B.16) and (B.11) at $y=y_{0}=0$, we find the relation between the constants

$$
A_{z}=B_{z}\left(\frac{\sqrt{\lambda_{z}}+k \kappa_{0, z}}{\sqrt{\lambda_{z}}-k \kappa_{0, z}}\right)
$$

At $y=y_{1}=1$ we get the equation that determines $m_{z}^{2}$,

$$
\lambda_{z}+k \sqrt{\lambda_{z}}\left(\kappa_{0 z}-\kappa_{1 z}\right) \epsilon_{\lambda}-k^{2} \kappa_{0 z} \kappa_{1 z}=0
$$

where

$$
\epsilon_{\lambda}=\frac{1+e^{-2 \sqrt{\lambda_{z}} k b_{0}}}{1-e^{-2 \sqrt{\lambda_{z}} k b_{0}}}
$$

While eq. (B.22) does not yield a closed-form solution for the masses in general, one can find analytic results in some approximations. Expanding $k \sqrt{\lambda_{z}} \simeq \frac{3 k^{2}}{2}-\frac{m_{z}^{2}}{3}$ for $m_{z}^{2} \ll k^{2}$ and ignoring the small exponential terms we can solve for the masses of the two light modes as

$$
m_{z}^{2} \simeq-\frac{2 \sigma_{0} k^{2}}{1-\frac{\sigma_{0}}{3}}, \frac{\sigma_{1} k^{2}}{1+\frac{\sigma_{1}}{3}}
$$

in agreement with eq. (4.15).

\section{References}

[1] P. Horava and E. Witten, "Eleven-Dimensional Supergravity on a Manifold with Boundary," Nucl. Phys. B 475, 94 (1996) [arXiv:hep-th/9603142]. "Heterotic and type I string dynamics from eleven dimensions," Nucl. Phys. B 460, 506 (1996) [arXiv:hep-th/9510209].

[2] L. Randall and R. Sundrum, "A large mass hierarchy from a small extra dimension," Phys. Rev. Lett. 83, 3370 (1999) [arXiv:hep-ph/9905221].

[3] W. D. Goldberger and M. B. Wise, "Modulus stabilization with bulk fields," Phys. Rev. Lett. 83, 4922 (1999) [arXiv:hep-ph/9907447]. 
[4] N. Arkani-Hamed, S. Dimopoulos, N. Kaloper and J. March-Russell, "Rapid asymmetric inflation and early cosmology in theories with sub-millimeter dimensions," Nucl. Phys. B 567, 189 (2000) [arXiv:hep-ph/9903224].

[5] N. Arkani-Hamed, S. Dimopoulos and G. R. Dvali, "The hierarchy problem and new dimensions at a millimeter," Phys. Lett. B 429, 263 (1998) [arXiv:hep-ph/9803315]; I. Antoniadis, N. Arkani-Hamed, S. Dimopoulos and G. R. Dvali, "New dimensions at a millimeter to a Fermi and superstrings at a TeV," Phys. Lett. B 436, 257 (1998) [arXiv:hep-ph/9804398].

[6] J. M. Cline, "Inflation from extra dimensions," Phys. Rev. D 61, 023513 (2000) [arXiv:hep-ph/9904495].

[7] R. Sundrum and C. M. Wells, "Warped Hybrid Inflation," [arXiv:0909.3254 [hep-ph]].

[8] A. Vilenkin, "Topological inflation," Phys. Rev. Lett. 72, 3137 (1994) [arXiv:hep-th/9402085].

[9] A. D. Linde and D. A. Linde, "Topological defects as seeds for eternal inflation," Phys. Rev. D 50, 2456 (1994) [arXiv:hep-th/9402115].

[10] R. C. Myers, "New Dimensions For Old Strings," Phys. Lett. B 199, 371 (1987).

[11] E. Kiritsis, C. Kounnas and D. Lust, "A Large class of new gravitational and axionic backgrounds for four-dimensional superstrings," Int. J. Mod. Phys. A 9, 1361 (1994) [arXiv:hep-th/9308124].

[12] J. Polchinski, "String Theory, Vol. 1, An Introduction to the Bosonic String," Cambridge University Press (2000); see eqs. (3.7.20) and (3.7.25).

[13] O. DeWolfe, D. Z. Freedman, S. S. Gubser and A. Karch, "Modeling the fifth dimension with scalars and gravity," Phys. Rev. D 62, 046008 (2000) [arXiv:hep-th/9909134].

[14] P. Breitenlohner and D. Z. Freedman, "Stability In Gauged Extended Supergravity," Annals Phys. 144, 249 (1982); "Positive Energy In Anti-De Sitter Backgrounds And Gauged Extended Supergravity," Phys. Lett. B 115, 197 (1982).

[15] P. Kanti, S. Lee and K. Olive, "Stable, time dependent, exact solutions for brane models with a bulk scalar field", Phys. Rev. D 67024037 (2003) [arXiv:hep-ph/0209036].

[16] N. Kaloper, "Bent domain walls as brane worlds," Phys. Rev. D60, 123506 (1999). [arXiv:hep-th/9905210].

[17] J. M. Cline and H. Firouzjahi, "Brane world cosmology of modulus stabilization with a bulk scalar field," Phys.Rev.D 64023505 (2001), [arXiv:hep-ph/0005235].

[18] D. Langlois and M Rodríguez-Martínez, "Brane cosmology with a bulk scalar field, " Phys. Rev. D 64123507 (2001), [arXiv:hep-th/0106245].

[19] K. Koyama and K. Takahashi, "Primordial fluctuations in bulk inflaton model, " Phys. Rev. D 67103503 (2003). [arXiv:hep-th/0301165].

[20] K. Koyama and K. Takahashi, "Exactly solvable model for cosmological perturbations in dilatonic brane worlds," Phys. Rev. D 68103512 (2003), [arXiv:hep-th/0307073].

[21] C. Csaki, M. L. Graesser and G. D. Kribs, "Radion dynamics and electroweak physics," Phys. Rev. D 63, 065002 (2001) [arXiv:hep-th/0008151]. 
[22] J. M. Cline and H. Firouzjahi, "Five-dimensional warped cosmological solutions with radius stabilization by a bulk scalar," Phys. Lett. B 495,271 (2000) [arXiv:hep-th/0008185].

[23] C. Csaki, M. Graesser, L. Randall and J. Terning, "Cosmology of brane models with radion stabilization" Phys.Rev. D 62045015 (2000). [arXiv:hep-ph/9911406].

[24] L. Kofman, J. Martin and M. Peloso, "Exact identification of the radion and its coupling to the observable sector," Phys. Rev. D 70, 085015 (2004) [arXiv:hep-ph/0401189].

[25] V. F. Mukhanov, "Gravitational instability of the universe filled with a scalar field", JETP Lett. 41, 493 (1985) [Pisma Zh. Eksp. Teor. Fiz. 41, 402 (1985)].

[26] M. Sasaki, "Large Scale Quantum Fluctuations In The Inflationary Universe," Prog. Theor. Phys. 76, 1036 (1986).

[27] P. Brax, C. van de Bruck, A.C. Davis, and C.S. Rhodes, "Cosmological evolution of brane world moduli," Phys. Rev. D 67, 023512 (2003) [arXiv: hep-th/0209158].

[28] J. Lesgourgues and L. Sorbo, "Goldberger-Wise variations: Stabilizing brane models with a bulk scalar," Phys. Rev. D 69, 84010 (2004) [arXiv: hep-th/0310007].

[29] J. J. Blanco-Pillado et al., "Racetrack inflation," JHEP 0411, 063 (2004) [arXiv:hep-th/0406230].

[30] L. Boubekeur and D. H. Lyth, "Hilltop inflation," JCAP 0507, 010 (2005) [arXiv:hep-ph/0502047].

[31] J. J. Blanco-Pillado et al., "Inflating in a better racetrack," JHEP 0609, 002 (2006) [arXiv:hep-th/0603129].

[32] Ph. Brax, S. C. Davis and M. Postma, "The Robustness of $n_{s}<0.95$ in Racetrack Inflation," JCAP 0802, 020 (2008) [arXiv:0712.0535 [hep-th]].

[33] K. Kohri, C. M. Lin and D. H. Lyth, "More hilltop inflation models," JCAP 0712, 004 (2007) [arXiv:0707.3826 [hep-ph]].

[34] C. P. Burgess, J. M. Cline and M. Postma, "Axionic D3-D7 Inflation," JHEP 0903, 058 (2009) [arXiv:0811.1503 [hep-th]].

[35] D. H. Lyth and A. Riotto, "Particle physics models of inflation and the cosmological density perturbation," Phys. Rept. 314, 1 (1999) [arXiv:hep-ph/9807278].

[36] D. H. Lyth, "What would we learn by detecting a gravitational wave signal in the cosmic microwave background anisotropy?," Phys. Rev. Lett. 78, 1861 (1997) [arXiv:hep-ph/9606387].

[37] G. Efstathiou and K. J. Mack, "The Lyth Bound Revisited," JCAP 0505, 008 (2005) [arXiv:astro-ph/0503360].

[38] L. McAllister, E. Silverstein and A. Westphal, "Gravity Waves and Linear Inflation from Axion Monodromy," [arXiv:0808.0706 [hep-th]].

[39] M. Cicoli, C. P. Burgess and F. Quevedo, "Fibre Inflation: Observable Gravity Waves from IIB String Compactifications," JCAP 0903, 013 (2009) [arXiv:0808.0691 [hep-th]].

[40] The Planck Blue Book, www.rssd.esa.int/SA/PLANCK/docs/Bluebook-ESA-SCI(2005)1_V2.pdf 
[41] N. Barnaby, J. R. Bond, Z. Huang and L. Kofman, "Preheating After Modular Inflation," [arXiv:0909.0503 [hep-th]].

[42] C. G. . Callan, J. A. Harvey and A. Strominger, "Supersymmetric string solitons," [arXiv:hep-th/9112030].

[43] J. Polchinski, "String Theory, Vol. 2, Superstring Theory and Beyond," Cambridge University Press (2000).

[44] I. R. Klebanov and M. J. Strassler, "Supergravity and a confining gauge theory: Duality cascades and $\chi$ SB-resolution of naked singularities," JHEP 0008, 052 (2000) [arXiv:hep-th/0007191].

[45] J. M. Maldacena and C. Nunez, "Towards the large N limit of pure N = 1 super Yang Mills," Phys. Rev. Lett. 86, 588 (2001) [arXiv:hep-th/0008001].

[46] A. H. Chamseddine and M. S. Volkov, "Non-Abelian solitons in N = 4 gauged supergravity and leading order string theory," Phys. Rev. D 57, 6242 (1998) [arXiv:hep-th/9711181]; "Non-Abelian BPS monopoles in N = 4 gauged supergravity," Phys. Rev. Lett. 79, 3343 (1997) [arXiv:hep-th/9707176].

[47] B. Greene, K. Hinterbichler, S. Judes and M. K. Parikh, "Smooth Initial Conditions from Weak Gravity," arXiv:0911.0693 [hep-th].

[48] A. R. Liddle, A. Mazumdar, and F. E. Schunck, "Assisted inflation," Phys. Rev. D58, 061301 (1998), [arXiv:astro-ph/9804177].

[49] J. M. Cline and J. Trudeau, in preparation. 